\title{
Los parámetros magnéticos como indicadores de contaminación por elementos mayores y plomo en suelos urbanos del Valle de Aburrá, Colombia
}

\author{
Alexander Sánchez-Duque ${ }^{1,3}$, Francisco Bautista ${ }^{1}$, Rubén Cejudo $^{2, *}$, \\ Avto Goguitchaichvili ${ }^{2}$ y Miguel Ángel Cervantes-Solano ${ }^{4}$
}

\author{
${ }^{1}$ Laboratorio Universitario de Geofísica Ambiental (LUGA), Centro de Investigaciones en Geografía Ambiental (CIGA), \\ Universidad Nacional Autónoma de México, Antigua Carretera a Pátzcuaro No. 8701, \\ Col. Ex-Hacienda de San José de la Huerta, C.P. 58190, Morelia, Mich., México. \\ ${ }^{2}$ Laboratorio Universitario de Geofísica Ambiental (LUGA), Instituto de Geofísica Unidad Michoacán (IGUM), \\ Universidad Nacional Autónoma de México, Antigua Carretera a Pátzcuaro No. 8701, \\ Col. Ex-Hacienda de San José de la Huerta, C.P. 58190, Morelia, Mich., México. \\ ${ }^{3}$ Adscripción actual: Tecnológico Nacional de México, campus Valle de Morelia, \\ km 6.5 Carretera Morelia-Salamanca, C.P. 58100, Morelia, Mich., México. \\ ${ }^{4}$ Escuela Nacional de Estudios Superiores Unidad Morelia, \\ Antigua Carretera a Pátzcuaro 8701, C.P. 58190, Morelia, Mich., México. \\ *ruben@igeofisica.unam.mx
}

\section{RESUMEN}

Presentamos un estudio de magnetismo ambiental con el objetivo de determinar la relación de los elementos mayores y el plomo con los parámetros magnéticos en suelos urbanos del Área Metropolitana de Valle de Aburrá (AMVA), Colombia. El estudio se realizó con la finalidad de establecer indicadores magnéticos que permitan estimar la concentración de minerales magnéticos, de elementos mayores y de $\mathrm{Pb}$; así como ubicar espacialmente las áreas de mayor contaminación en la zona urbana del AMVA. El estudio se realizó a partir de 83 muestras de suelo urbano, las cuales fueron sujetas a un análisis magnético; se determinó la concentración de los elementos mayores $\left(\mathrm{Al}_{2} \mathrm{O}_{3}, \mathrm{Fe}_{2} \mathrm{O}_{3}\right.$ y $\mathrm{MnO}$ ) y $\mathrm{Pb}$ por medio de fluorescencia de Rayos $\mathrm{X}$ de dispersión de energía. Se encontró una variación en la distribución del material magnético en el suelo urbano, el cual contiene una mezcla de minerales magnéticos de baja coercitividad o magnetita de origen natural y antrópico. Se detectaron partículas de tamaño superparamagnético en concentraciones medias y bajas en los suelos de baja emisión de partículas antrópicas, lo que sugiere un origen natural. Por otra parte, mediante la combinación de los parámetros magnéticos y el comportamiento de curvas termomagnéticas se encontraron suelos con concentración alta de mineral magnético de origen antrópico. Las concentraciones de $\mathrm{Al}_{2} \mathrm{O}_{3}$ y $\mathrm{Fe}_{2} \mathrm{O}_{3}$ son altas y similares en la mayor parte de los suelos urbanos. Los usos de suelo residencial y de espacio público que tienen pocas fuentes de emisión de partículas antrópicas presentaron un contenido bajo de $\mathrm{Pb}$, mientras que los usos de suelo de tipo comercial e industrial, que muestran mayor actividad antrópica (tráfico vehicular), presentaron una concentración de $\mathrm{Pb}$ mayor. $\mathrm{El}$ suelo de los espacios públicos con baja emisión de partículas antró- picas fue empleado para determinar el valor de referencia para cada elemento y parámetro magnético. El análisis estadístico mostró que los suelos del AMVA tienen una relación directamente proporcional entre el material magnético y la concentración de $\mathrm{Pb}$ e inversamente proporcional con la concentración de $\mathrm{Al}_{2} \mathrm{O}_{3}, \mathrm{Fe}_{2} \mathrm{O}_{3}$ y MnO. El modelo matemático que predice la concentración de elementos a partir de parámetros magnéticos tiene una precisión del $63 \%$ y se encontró que el área que ocupan las zonas de acumulación de material magnético y contenido de $\mathrm{Pb}$ es de alrededor de $46 \mathrm{~km}^{2}$, lo cual representa el $25 \%$ de la zona urbana estudiada y es el área probable de riesgo a la salud por acumulación de material contaminante.

Palabras clave: parámetros magnéticos; suelo; contaminación; plomo; elementos mayores; valores de referencia; distribución espacial; Valle de Aburrá; Colombia.

\section{ABSTRACT}

We present a study of environmental magnetism in order to determine the relationship between magnetic parameters and heavy metals in urban soils of the Metropolitan Area of Valle de Aburrá (MAVA), Colombia, in order to establish magnetic indicators that allow estimating concentrations of magnetic minerals, mayor elements, and $\mathrm{Pb}$, and to determine the area of their accumulations in the urban zone of the Metropolitan Area of Valle de Aburrá. The study was carried out on 83 samples of topsoil, which were subject to magnetic analyses. The concentration of mayor elements $\left(\mathrm{Al}_{2} \mathrm{O}_{3}, \mathrm{Fe}_{2} \mathrm{O}_{3}\right.$ y $\left.\mathrm{MnO}\right)$ and $\mathrm{Pb}$ was determined by energy dispersive $\mathrm{X}$ Ray Fluorescence. A variation of magnetic material in the urban soils was
\end{abstract}


found, which contain a mixture of magnetic minerals of low coercivity or natural magnetite of anthropogenic origin. Particles of superparamagnetic size were detected at medium and low concentrations in soils with low anthropic activity, suggesting a natural origin. On the other hand, by the combination of magnetic parameters and thermomagnetic curves we found high concentrations of magnetic minerals of anthropic origin in the soils. The concentrations of $\mathrm{Al}_{2} \mathrm{O}_{3}$ y $\mathrm{Fe}_{2} \mathrm{O}_{3}$ are high and similar in the urban soil. Soils from areas of residential and public space use, which have few emission sources of anthropogenic particles, showed low concentration of $\mathrm{Pb}$. On the other hand, soils from areas of industrial and commercial use, with high anthropogenic activity (vehicular traffic), showed high concentration of $\mathrm{Pb}$. Soils from public space areas with low anthropic activity were employed to determine the reference threshold value for each element and magnetic parameter. The statistical analyses showed that the magnetic mineral content in the MAVA soils has a direct proportional relationship with the $\mathrm{Pb}$ concentration, and an inverse proportional relationship with the $\mathrm{Al}_{2} \mathrm{O}_{3}, \mathrm{Fe}_{2} \mathrm{O}_{3}$ and $\mathrm{MnO}$ concentration. We developed a mathematic model that estimates the concentration of elements from magnetic parameters with a precision of $63 \%$. High accumulation of magnetic minerals and high $\mathrm{Pb}$ concentration occur in an area of $46 \mathrm{~km}^{2}$, which represents $25 \%$ of the urban zone, and is the probable zone of health risk by accumulation of polluted material.

Key words: Magnetic parameters; soil; contamination; lead; major elements; threshold value; spatial distribution; Aburrá valley, Colombia.

\section{INTRODUCCIÓN}

El crecimiento de las zonas urbanas incrementa el número de fuentes emisoras (emisiones vehiculares o industriales) de partículas, lo cual provoca una contaminación del entorno como, por ejemplo, la disminución de la calidad del aire o el incremento de elementos en el suelo a niveles tóxicos, problema que se acentúa en ciudades de países en vía de desarrollo, en donde la expansión urbana suelen tener poca planificación, suele estar mal administrada o cuenta con escasez de recursos financieros para el mantenimiento de la infraestructura urbana (Rodríguez-Eugenio et al., 2019).

Actualmente hay un interés por conocer la concentración de metales pesados en suelos urbanos, debido a que su alta concentración puede representar un peligro para la población. Los metales pesados no son biodegradables, por lo que pueden ser acumulados inadvertidamente en el suelo a niveles tóxicos (Micó et al., 2006; Hernández et al., 2007), para luego ingresar otras esferas ambientales y entrar a las redes tróficas hasta llegar a convertirse en un problema de salud.

Una de las funciones del suelo es ser un sumidero de contaminantes, actuando como un amortiguador natural que controla el transporte de elementos químicos y sustancias a la atmósfera, hidrosfera y biosfera (Kabata-Pendias, 2010). Sin embargo, hasta ahora la contaminación del suelo por metales pesados aún no recibe la misma atención que la contaminación del aire y del agua (Cairney y Hobson, 1998).

Los elementos mayores y metales pesados se encuentran de manera natural en el suelo, en concentraciones generalmente no perjudiciales para las diferentes formas de vida. Estas concentraciones pueden modificarse de varias maneras por la actividad antrópica, por ejemplo, por la eliminación de desechos sólidos domésticos e industriales, por el depósito de partículas provenientes de la atmósfera por vía seca (asentamiento gravitacional) y vía húmeda (por acción de la lluvia, nieve, niebla, nubes que transitan rasantes a la superficie del suelo), por infiltración por aguas contaminadas (Harrison, 2006).

Para el caso de los metales pesados en el suelo, estos pueden permanecer relativamente inmóviles durante decenas o cientos de años, por ejemplo, el plomo, el cual es un elemento ampliamente utilizado en la industria. Este metal es de un gran riesgo para la salud de la población, debido a que daña el cerebro, los riñones y puede causar la muerte (Londoño-Franco et al., 2016).

Algunas investigaciones han probado que los parámetros magnéticos de muestras ambientales (suelo, polvo urbano, material botánico), tales como la susceptibilidad magnética $(\chi)$ y la magnetización remanente isotérmica a saturación (MRIS), se encuentran relacionadas con la concentración de metales pesados y, en algunos casos, por medio de variaciones de los parámetros magnéticos se puede asociar el origen del material magnético a diferentes actividades (Heller et al., 1998; Jordanova et al., 2003; Spiteri et al., 2005; Lu y Bai, 2006; Karimi et al., 2011; Wang, 2013; Xia et al., 2014; Pérez-Martínez y Romero, 2015; Cejudo-Ruíz et al., 2015; Liu et al., 2016).

La calibración correcta entre los parámetros magnéticos y la concentración de metales ( $\mathrm{Pb}, \mathrm{Al}, \mathrm{Fe}$ y $\mathrm{Mn}$ ) en el suelo mejorará la interpretación de resultados, lo que permitirá determinar las áreas con peligrosidad potencial. Los metales pesados y elementos mayores se encuentran de forma natural en la corteza terrestre y su concentración difiere de un lugar a otro, por lo que es necesario establecer un nivel de fondo o valor de referencia para cada elemento que permita identificar si un sitio representa un riesgo, esto es, si el valor de concentración es mayor al valor de fondo o referencia (Hanesch et al., 2007; Jordanova et al., 2008, Brus et al., 2009; Rueda et al., 2011).

Los objetivos de esta investigación fueron: a) determinar la relación entre la concentración de elementos mayores y el plomo con los parámetros magnéticos; $\mathrm{y} b$ ) identificar las zonas de peligrosidad potencial por acumulación de elementos mayores y plomo en el Área Metropolitana de Valle de Aburrá, Colombia.

\section{MATERIALES Y MÉTODOS}

\section{Área de estudio}

El Valle de Aburrá se extiende desde las proximidades del nacimiento del río Medellín, en el extremo suroccidental, hasta la zona central del departamento de Antioquia (Colombia). La ciudad de Medellín es el principal centro urbano (ciudad núcleo) del valle, y junto con otros nueve municipios (Barbosa, Girardota, Copacabana, Bello, Itagüí, La Estrella, Sabaneta, Envigado y Caldas) integran el Área Metropolitana del Valle de Aburrá (AMVA). El AMVA tiene una superficie de $1157 \mathrm{~km}^{2}$, una población de 3.8 millones de habitantes, y una densidad de 22,681 habitantes $/ \mathrm{km}^{2}$. El municipio de Medellín contiene la mayor parte de la zona urbana, la cual tiene una superficie de $183 \mathrm{~km}^{2}$ (CGM, 2015; Mejía-Echeverry et al., 2018) (Figura 1)

El AMVA se extiende longitudinalmente sobre el eje natural del río Medellín enmarcado en una topografía irregular y pendiente, con altitudes que oscilan entre 1300 y 2800 m s.n.m. Las cordilleras que lo rodean dan lugar a la formación de diversos microclimas, en donde se registra una precipitación anual de $1400 \mathrm{~mm}$ a $3000 \mathrm{~mm}$ durante los meses de abril y de octubre (CGM, 2015). La temperatura media anual de la ciudad de Medellín es de $24^{\circ} \mathrm{C}$ (Alcaldía de Medellín, 2011). El Valle de Aburrá se encuentra sobre rocas metamórficas (esquistos, anfibolitas, migmatitas y gneises) del Paleozoico, suprayacidas por rocas ígneas (granodioritas, dunitas, gabros y basaltos), cubiertas por rocas volcano-sedimentarias del Cuaternario y depósitos de origen aluvial y de vertiente del Holoceno, con desarrollo de suelos de varios tipos (UN/Medellín et al., 2007; Lince-Prada y Rave-Herrera, 2010).

\section{Muestreo}

El estudio comprendió la zona urbana del AMVA, la cual contiene una variedad de usos de suelo (Tabla 1; MMA, 2012). Se recolectaron 


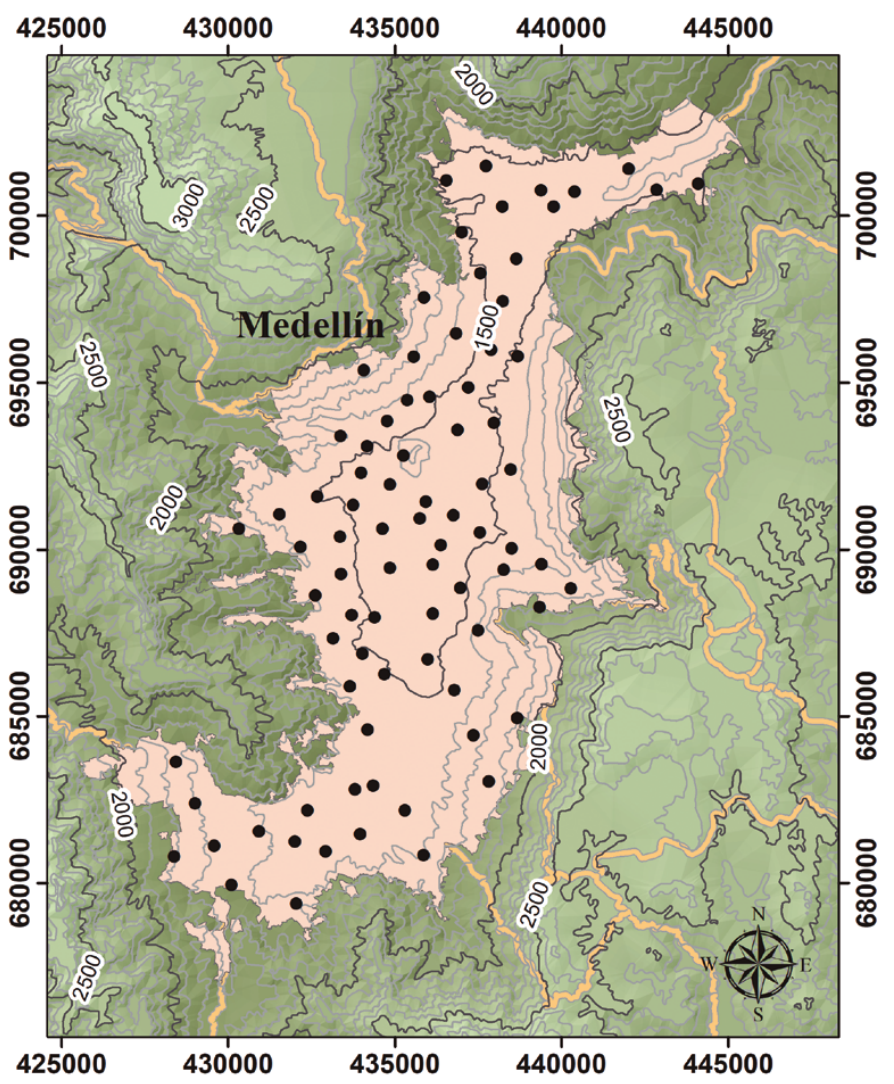

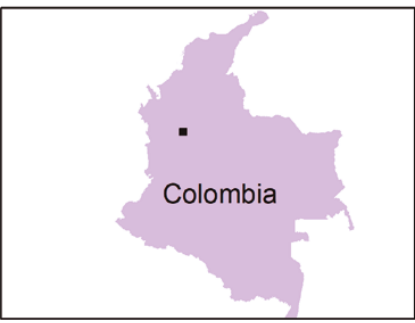

Leyenda

- Sitios

Zona urbana

Símbolos convencionales

Carretera

Fecha de muestreo

AMVA--------------Enero de 2014

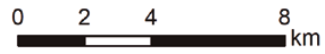

Referencia espacial

Elipsoide.....................WGS84 1984 Proyección..........Transversal Mercantor Zona.....................................18 Norte Datum........................WS 1984 Meridiano Central................... $75^{\circ}$ Oeste Longitud de referencia................ $0^{\circ}$ Norte Unidades......................................... Metro

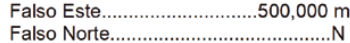

Figura 1. Localización de sitios de muestreo en la zona urbana del Área Metropolitana Valle de Aburrá, Colombia.

83 muestras de suelo superficial mediante un muestreo sistemático con separación entre muestra de $1.4 \mathrm{~km}$ aproximadamente. Los datos de georreferencia fueron tomados con un receptor GPS bajo el sistema de coordenadas UTM Zona 18 Norte con datum WGS84 y fecha. Cada sitio fue registrado con la clasificación del uso de suelo de acuerdo al plan de ordenamiento territorial de la ciudad de Medellín (Tabla 1) (DAPM, 2014).

Se realizó un muestreo sistemático, para lo cual la muestra se extrajo con un cilindro de PVC o aluminio de $5 \mathrm{~cm}$ de diámetro y 5 $\mathrm{cm}$ de profundidad. En el punto de extracción se retiraron las plantas y otros residuos no vegetales, el material fue depositado en una bolsa de polipropileno con cierre y con su respectiva etiqueta, con los datos de identificación: sitio, coordenadas geográficas, uso de suelo y fecha. La recolección de muestras se llevó a cabo durante la segunda y tercera semana de enero de 2014.

Se hizo una extracción de cuatro muestras de suelo a $20 \mathrm{~cm}$ de profundidad (suelo de control) en la zona periurbana de la AMVA, con la finalidad de tener valores de referencia para parámetros magnéticos y concentración de elementos que pudieran ser contrastados con los valores de suelos en áreas con uso de espacio público. El área de recolección del suelo de control mostró muy pocas fuentes de emisiones antrópicas.

Las muestras se prepararon bajo los procedimientos descritos en la norma NOM-021-RECNAT-2000 (SEMARNAT, 2002), los cuales consistieron en el secado a temperatura inferior a $35^{\circ} \mathrm{C}$, con humedad relativa de 30 a $70 \%$ durante dos semanas. Posteriormente se destruyeron los agregados de suelo y se retiraron restos visibles de material orgánico y fragmentos de rocas. El suelo se tamizó con ayuda de una malla de plástico con abertura de $1 \mathrm{~mm}$ (malla 18), y el material obtenido se encapsuló en cubos de acrílico de $8 \mathrm{~cm}^{3}$ para los análisis magnéticos y para los estudios geoquímicos.

\section{Análisis magnético}

Antes de realizar el análisis magnético, a cada muestra encapsulada se le determinó la densidad $\left(\rho \mathrm{en} \mathrm{kg} \cdot \mathrm{m}^{-3}\right)$. Las distintas mediciones magnéticas permiten identificar en la muestra minerales magnéticos, tipo de mineral magnético predominante, tamaño de grano magnético y, por medio de la combinación de los parámetros magnéticos de susceptibilidad magnética y magnetización remanente, se pueden establecer indicios del origen antrópico o natural del material magnético (Dearing, 1999: Evans y Heller, 2003).

Las mediciones de susceptibilidad magnética con baja $\left(\kappa_{\mathrm{lf}}: 470 \mathrm{~Hz}\right)$ y alta frecuencia $\left(\kappa_{\mathrm{hf}}: 4700 \mathrm{~Hz}\right)$, se realizaron empleando un susceptibilímetro magnético Bartington con sensor de frecuencia dual MS2B (Dearing, 1999).

Se determinaron: a) el parámetro de susceptibilidad específica $\left(\chi_{\mathrm{If}}=\kappa_{\mathrm{lf}} / \rho\right.$, donde $\kappa_{\mathrm{If}}$ es la susceptibilidad magnética de baja frecuencia, $y, \rho$ es la densidad en unidades de $\mathrm{kg} \cdot \mathrm{m}^{-3}$ ) que es dependiente de la concentración de minerales que hay en la muestra; b) el porcentaje de la susceptibilidad dependiente de la frecuencia, $\chi_{\mathrm{df}} \%\left(\left(\chi_{\mathrm{df}} \%=\left(\kappa_{\mathrm{lf}}-\kappa_{\mathrm{hf}}\right) /\right.\right.$ $\left.\kappa_{\mathrm{lf}}{ }^{\star} 100\right)$ que es un parámetro magnético utilizado para estimar la concentración de minerales ferromagnéticos de grano ultrafino (diámetro $<30 \mathrm{~nm}$ ) superparamagnéticos (SP), a partir del cual se puede evaluar la concentración en baja $\left(\chi_{\mathrm{df}} \%<2 ;\right)$, media $\left(\chi_{\mathrm{df}} \%: 2-10\right)$, alta $\left(\chi_{\mathrm{df}} \%\right.$ : $10-14)$ o muy alta $\left(\chi_{\mathrm{df}} \%>14\right)$ (Dearing, 1999; Evans y Heller, 2003).

A cada muestra encapsulada de suelo se le indujo una magnetización remanente mediante la aplicación de un pulso magnético unidireccional de $0.2,0.5$ y $0.7 \mathrm{~T}$, usando un magnetizador de pulsos 
Tabla 1. Uso de suelo para el Área Metropolitana del Valle de Aburrá, Colombia.

\begin{tabular}{|c|c|c|}
\hline Uso de suelo & Sigla & Descripción \\
\hline $\begin{array}{l}\text { Áreas y corredores de } \\
\text { alta mixtura }\end{array}$ & ACAM & $\begin{array}{l}\text { Predomina el uso del suelo en función de las actividades económicas y la prestación de servicios públicos, con menor } \\
\text { proporción del uso residencial. Incluye las subcategorías: Comercio y servicios (ACAM-CS); industrial (ACAM-I). }\end{array}$ \\
\hline Espacio público & EP & $\begin{array}{l}\text { Bienes de uso público y áreas libres (públicas o privadas) de carácter permanente, destinados a la recreación, } \\
\text { esparcimiento, ocio y encuentro ciudadano. }\end{array}$ \\
\hline
\end{tabular}

ASC IM10-30. Luego de la aplicación de cada campo se midió la magnetización remanente isotérmica $\left(\mathrm{MRI}_{\mathrm{x}}\right.$, donde $\mathrm{x}$ es el campo aplicado) inducida con ayuda de un magnetómetro de giro Molspin LTD. La magnetización remanente adquirida a $0.7 \mathrm{~T}$ fue considerada como la magnetización remanente isotérmica de saturación (MRIS), cuyo valor está asociado con la concentración relativa de material magnético de baja coercitividad o ferrimagnético (Evans y Heller, 2003; Liu et al., 2012).

Una vez adquirida la MRIS se indujo una magnetización en dirección opuesta a la dirección normal mediante la aplicación del pulso magnético de $0.2 \mathrm{~T}\left(\mathrm{MRI}_{-0.2}\right)$ para determinar el cociente $\mathrm{S}_{-0.2}\left(\mathrm{~S}_{-0.2}=\mathrm{MRI}_{-0.2} / \mathrm{MRIS}\right)$. Si el valor de $S_{-0.2}<0.7$, los minerales de alta coercitividad (antiferromagnéticos) predominan en la muestra. Por otro lado, si los valore de $S_{-0.2}$ se encuentran entre 0.7 a 1.0, predominan en la muestra los minerales de baja coercitividad (ferrimagnéticos) (Thompson y Oldfield, 1986, Evans y Heller, 2003).

Para identificar los portadores magnéticos por medio de las fases magnéticas o temperatura de Curie, se midió la variación de la susceptibilidad magnética $(\kappa)$ con el incremento continuo de la temperatura $(\mathrm{T})$ (curva termomagnética, $\kappa \mathrm{T}$ ). Se utilizó un susceptibilímetro magnético Bartington MS2 equipado con horno de alta sensibilidad $\left(2 \times 10^{-8} \mathrm{SI}\right)$ a una razón de calentamiento de $10^{\circ} \mathrm{C}$. Una muestra de $2 \mathrm{~g}$ fue calentada desde temperatura ambiente hasta alcanzar $700{ }^{\circ} \mathrm{C} \mathrm{y}$ posteriormente fue enfriada al mismo ritmo. La identificación de las fases magnéticas se hizo por medio del método diferencial descrito por Tauxe et al., (2018).

\section{Análisis geoquímicos}

La determinación de los elementos mayores $\left(\mathrm{Al}_{2} \mathrm{O}_{3}, \mathrm{Fe}_{2} \mathrm{O}_{3}\right.$ y $\left.\mathrm{MnO}\right)$ y $\mathrm{Pb}$ en las muestras de suelo se realizaron con un espectrómetro Xenemetrix (X-Calibur) de Fluorescencia de Rayos X de Dispersión de Energía (EDXRF en inglés), el cual tiene capacidad de 8 pellets. Los rayos $\mathrm{X}$ se generan en un tubo con ánodo $\mathrm{Rh} / \mathrm{Ag} / \mathrm{Mo} / \mathrm{W} / \mathrm{Pd}$ con una energía de $40 \mathrm{kV}, 18 \mathrm{~W}$; el sensor de rayos $\mathrm{X}$ es de silicón SDD, capaz de detectar concentraciones en ppm y porcentuales con precisión de $0.1 \%$ a temperatura ambiente (Xenemetrix, 2020).

La muestra para estudio geoquímico fue pulverizada y tamizada por una malla de № $230(63 \mu \mathrm{m})$. La muestra para FRX fue preparada con una mezcla de $6 \mathrm{~g}$ de material tamizado y $5 \mathrm{~g}$ de cera $\mathrm{C}$ de Hoechst como aglutinante, la mezcla fue colocada en una prensa hidráulica manual a $10 \mathrm{t}$ para obtener un pellet de $2 \mathrm{~cm}$ de diámetro.

Las mediciones de elementos mayores $\left(\mathrm{Al}_{2} \mathrm{O}_{3}, \mathrm{Fe}_{2} \mathrm{O}_{3}\right.$ y $\left.\mathrm{MnO}\right)$ y $\mathrm{Pb}$ se hicieron con un filtro de $\mathrm{Rh}$ y Ti, a $40 \mathrm{kV}$ por $45 \mathrm{~s}$ y por quintuplicado. La curva de calibración fue obtenida con los estándares de
FRX de la serie IGL, cuyo coeficiente de correlación (CC) fue de 0.98 (Lozano y Bernal, 2005).

Los análisis geoquímicos se hicieron en el Laboratorio Universitario de Geofísica Ambiental del Instituto de Geofísica Unidad Michoacán de la Universidad Nacional Autónoma de México, Campus Morelia.

\section{Análisis de datos}

Se realizó un análisis de estadística descriptiva de los datos. Los parámetros utilizados para determinar la correlación y modelación espacial ( $\chi$, MRIS, $\mathrm{Al}_{2} \mathrm{O}_{3}, \mathrm{Fe}_{2} \mathrm{O}_{3}$ y $\mathrm{MnO}$ ) fueron sujetos a un análisis exploratorio de datos y un análisis geoespacial. En el análisis exploratorio de datos se identificaron los valores atípicos y se aplicó la prueba de normalidad Shapiro-Wilks con un nivel de significancia del $5 \%$ (valor p por debajo de 0.05 rechaza la hipótesis de normalidad).

Posteriormente se realizó un análisis multivariado que incluyó: análisis de componentes principales $(\mathrm{ACP})$ y análisis clúster $(\mathrm{AC})$ para clasificar las muestras en dos grupos: el Grupo 1 formado por muestras que no presentan relación entre parámetros magnéticos y geoquímicos, y el Grupo 2 para muestras que presentan relación entre los parámetros magnéticos y geoquímicos. Se usó el Grupo 2 para identificar el uso de suelo con los valores más bajos de elementos y parámetros magnéticos, con la finalidad de definir un valor de referencia. Se usó un análisis de varianza simple (ANOVA) para identificar el uso de suelo con los valores más bajo, y el valor de referencia (VR) se definió como el valor de la mediana más el valor de la desviación estándar $\left(\mathrm{VR}=\mathrm{X}_{\text {mediana }}+\sigma\right)$ (Rueda-Saa et al., 2011).

Para obtener un modelo matemático que permita estimar la concentración de elementos mayores y $\mathrm{Pb}$ a partir de mediciones magnéticas, se llevó al cabo un análisis de correlación lineal y no lineal para el Grupo 2.

El análisis espacial de los parámetros $\chi$, MRIS, $\mathrm{Al}_{2} \mathrm{O}_{3}, \mathrm{Fe}_{2} \mathrm{O}_{3}$ y $\mathrm{MnO}$ fue realizado bajo los criterios recomendados por Oliver y Webster $(2014,2015)$ con la finalidad de determinar la distribución espacial de cada parámetro, análisis estructural y modelo de interpolación; para este estudio se empleó la interpolación Kriging Ordinario.

El análisis estructural permitió comprobar la existencia de correlación espacial a través del cálculo del variograma experimental, el cual utiliza la función de correlación espacial de semivarianza a partir de la Ecuación 1:

$$
\mathrm{Y}(\mathrm{h})=\frac{1}{2 \mathrm{n}} \sum_{\mathrm{i}=1}^{\mathrm{n}}\left[\mathrm{Z}\left(\mathrm{x}_{\mathrm{i}}\right)-\mathrm{Z}\left(\mathrm{x}_{\mathrm{i}}+\mathrm{h}\right)\right]^{2}
$$

Donde $\mathrm{Y}(\mathrm{h})$ es el valor de la semivarianza estimada o variograma experimental para todos los pares de puntos separados una distancia $h$; 

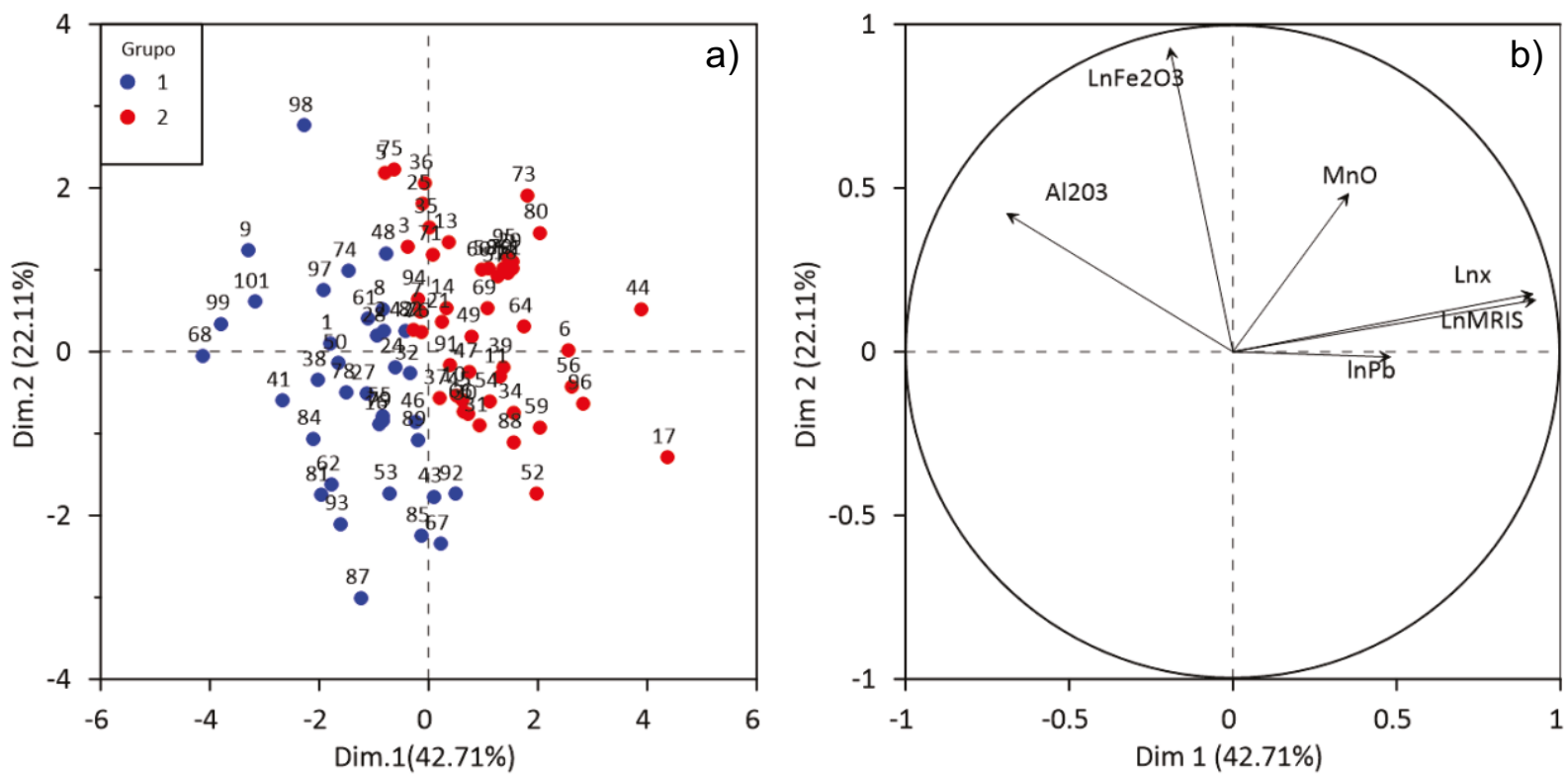

Figura 2. a) Proyecciones de las muestras clasificadas en grupos (Grupo 1 para muestras sin relación entre parámetros magnéticos y geoquímicos y el Grupo 2 para muestras con relación entre los parámetros magnéticos y geoquímicos) en el plano conformado por la primera y segunda componente del análisis de componentes principales y b) círculo de correlaciones de las concentraciones de elementos y parámetros magnéticos proyectados en las dos primeras componentes principales.

$\mathrm{Z}\left(\mathrm{x}_{\mathrm{i}}\right)$ es el valor de la variable en el sitio $\mathrm{x}$ i-ésimo punto; $\mathrm{Z}\left(\mathrm{x}_{\mathrm{i}}+\mathrm{h}\right)$ es el valor de la variable seleccionada en el punto $\mathrm{x}_{\mathrm{i}}$ separado por una distancia $h$ del punto i; $n$ es el número de pares de observaciones separadas por una distancia h llamada intervalo o lag.

Los resultados permitieron establecer un modelo de variograma teórico al variograma experimental, y de esta forma se obtuvieron la suma de cuadrados del error (SCE) y el coeficiente de determinación $\left(\mathrm{R}^{2}\right)$, la semivarianza estructural $\left(\mathrm{C}_{1}\right)$, semivarianza del nugget o efecto pepita, $\left(\mathrm{C}_{0}\right)$, sill o meseta $\left(\mathrm{C}_{0}+\mathrm{C}_{1}\right)$, y rango o alcance $(a)$, que permiten identificar el modelo de variograma más adecuado para la interpolación de los datos (Isaaks y Srivastava, 1990; Webster y Oliver, 1990; Chilès y Delfiner, 2012). Para evaluar el modelo de interpolación se empleó el método de validación cruzada.

Para el análisis estadístico se utilizó el software R, el análisis estructural y la predicción espacial se realizó con el software GS+ (Gamma Design Software, 2014) y la representación espacial con el software ArcGIS 9.1 (ESRI, 2005) con datos de proyección UTM Zona 18 Norte, elipsoide y Datum horizontal del World Geodetic System 84 (WGS84).

\section{RESULTADOS Y DISCUSIÓN}

La formación de dos grupos de muestras: con y sin relación entre parámetros magnéticos y elementos mayores

El análisis de componentes principales permitió identificar las variables que mantienen similitudes, usando los componentes 1 y 2 , la información quedó representada en un $64.82 \%$. La suma de los $\cos ^{2}$ de las variables representados con la dimensión 1 y 2 mostraron que, tanto el $\mathrm{Al}_{2} \mathrm{O}_{3}, \mathrm{Fe}_{2} \mathrm{O}_{3}, \chi_{\text {lf }}$ y el MRIS quedan bien representados (suma $\cos ^{2} \geq 0.5$ ) en comparación con $\mathrm{MnO}$ y $\mathrm{Pb}\left(\right.$ suma $\left.\cos ^{2}<0.5\right)$ en el círculo de correlación (Figura 2).

En el plano principal se observan dos agrupaciones: el Grupo 1 reunió las muestras que presentaron poca o nula relación entre parámetros magnéticos y geoquímicos, el Grupo 2 reunión las muestras que presentaron una relación entre los parámetros magnéticos y geoquímicos (Figura 2). El círculo de correlaciones indica que hay una relación proporcional entre $\mathrm{Mn}, \mathrm{Pb}$, $\chi_{\text {lf }}$ y MRIS, mientras que $\mathrm{Al}_{2} \mathrm{O}_{3}$ mantiene una relación inversa con los parámetros magnéticos, y el contenido de $\mathrm{Fe}_{2} \mathrm{O}_{3}$ no mostró relación con los parámetros magnéticos (Figura 2).

La agrupación obtenida indicó que 31 sitios pertenecen al Grupo 1 (sin correlación) y 52 sitios pertenecen al Grupo 2 (con correlación).

\section{Los parámetros magnéticos}

Los suelos superficiales de la zona urbana mostraron valores de $\chi_{\text {If }}$ entre 0.23 a $4.39 \mu \mathrm{m}^{3} \cdot \mathrm{kg}^{-1}$, con una mediana de $0.78 \mu \mathrm{m}^{3} \cdot \mathrm{kg}^{-1}$; los valores de MRIS se encontraron entre 1.61 a $10.88 \mathrm{mAm}^{2} \cdot \mathrm{kg}^{-1}$ con valor de mediana de $5.81 \mathrm{mAm}^{2} \cdot \mathrm{kg}^{-1}$; los valores de $\chi_{\mathrm{df}} \%$ entre $0.12 \mathrm{a}$ $5.37 \%$ y S $_{-200}$ entre 0.56 a 0.97 .

Los suelos mostraron una concentración y tamaño de minerales magnéticos variable, con sitios con una concentración alta de material magnético de baja coercitividad (magnetita), los cuales mostraron valores de MRIS $\left(>2.13 \mathrm{mAm}^{2} \cdot \mathrm{kg}^{-1}\right)$ y $\chi_{\text {If }}\left(>0.38 \mu \mathrm{m}^{3} \cdot \mathrm{kg}^{-1}\right)$ mayores a los del suelo control, por lo que existe una contribución de material magnético de origen antrópico (Figura 3) (Thompson y Oldfield, 1986).

Los parámetros MRIS y $\chi_{\mathrm{If}}$ mostraron una relación lineal positiva entre ellos, por lo que es posible utilizar un solo parámetro para describir la presencia de material magnético en los suelos urbanos (Figura 3a).

Se detectaron partículas superparamagnéticas (SP, tamaño $<30 \mathrm{~nm}$ ) en los suelos de Medellín en concentraciones bajas y medias. Los suelos con concentraciones medias de SP correspondieron a los usos de suelo EP y ABM (Tabla 1; Tabla S1 del material suplementario), los cuales suelen tener poco tráfico vehicular y se encuentran lejos de áreas industriales, por lo que las emisiones de material contaminante es baja; su valor de susceptibilidad magnética fue bajo $\left(\chi_{\mathrm{lf}}<1.5 \mu \mathrm{m}^{3} \cdot \mathrm{kg}^{-1}\right)$ por lo que contienen poco material antrópico, por lo tanto, las partículas SP de estos usos de suelo son principalmente de origen edafológico o génesis del suelo (Figura 3b, Evans y Heller, 2003).

Los suelos exhibieron mayormente valores de $S_{-200}>0.7$, por lo que el material magnético fue de baja coercitividad. Este resultado 

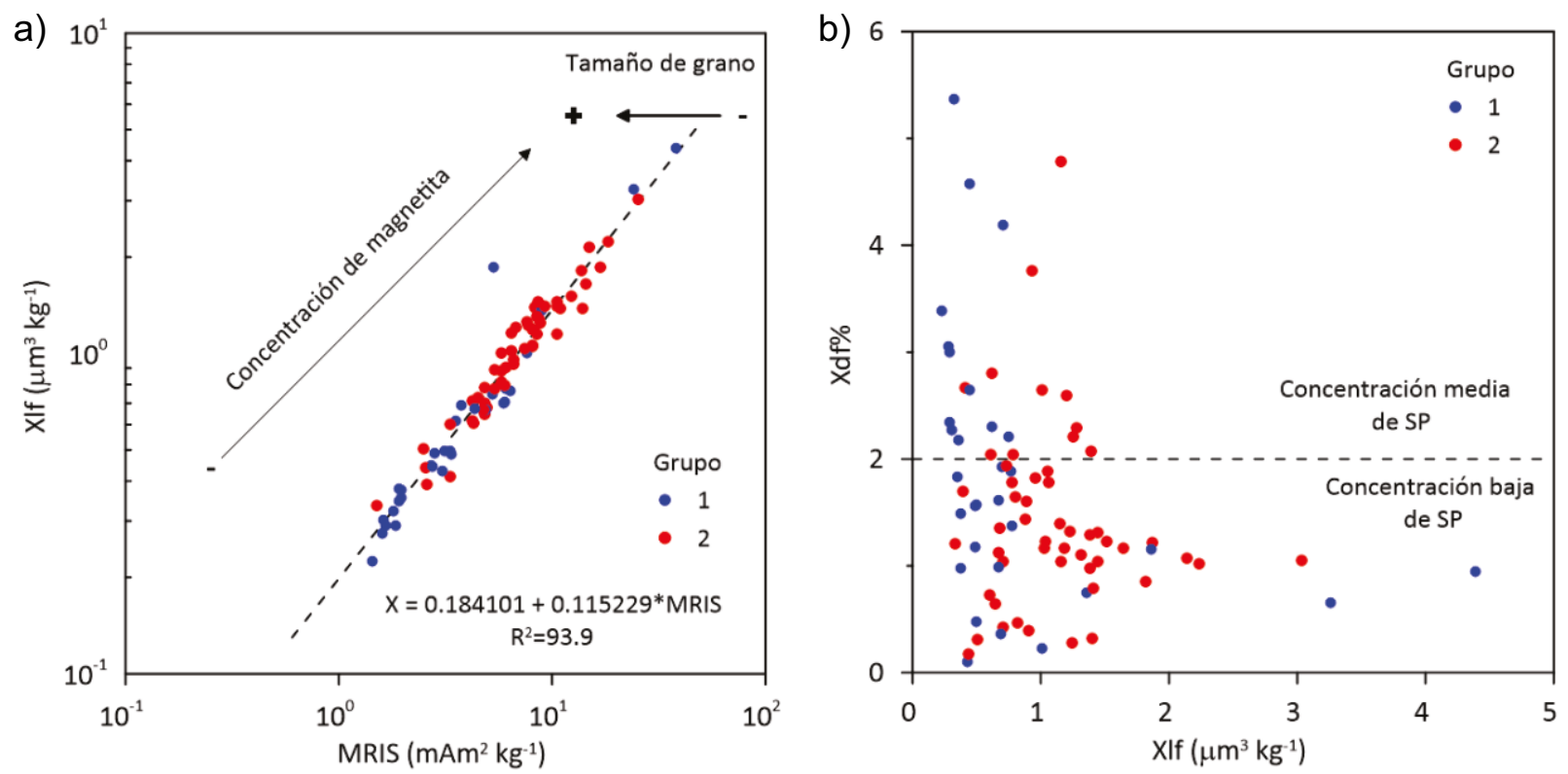

Figura 3. a) Relación entre la magnetización remanente isotérmica a saturación (MRIS) y la susceptibilidad específica ( $\chi_{\mathrm{lf}}$ ). Las muestras con bajo contenido de material magnético se encuentran en la parte inferior izquierda y las de mayor contenido se encuentran en la esquina superior derecha. b) Relación entre la $\chi_{\text {If }}$ y la susceptibilidad dependiente de la frecuencia $\left(\chi_{\mathrm{df}} \%\right)$ para muestras de suelo que indica la presencia y concentración de material superparamagnético (SP).

concuerda con la mineralogía magnética encontrada en las curvas termomagnéticas (Figura 4).

A partir del análisis de las curvas termomagnéticas, curvas kT se observa que tienen una sola fase magnética con una temperatura de Curie cercana a los $580^{\circ} \mathrm{C}$, compatible con titanomagnetita con contenido bajo en titanio, por lo que se puede establecer que este es el mineral magnético principal en los suelos superficiales de Medellín (Figura 4).

En algunos casos se observaron otras fases magnéticas menos significativas que indican la presencia de otros minerales magnéticos como la goethita $\left(350{ }^{\circ} \mathrm{C}\right)$ y magnetita metaestable $\left(450\right.$ a $\left.500{ }^{\circ} \mathrm{C}\right)$ probablemente de origen antrópico, ya que se observa un incremento abrupto de la susceptibilidad magnética durante el calentamiento entre 450 a $500{ }^{\circ} \mathrm{C}$ (pico de Hopkinson), el cual se ha observado en muestras adicionadas con material magnético ultrafino con contenido de hierro proveniente de emisiones vehiculares (Sánchez-Duque et al., 2015).

\section{Los elementos mayores y el plomo}

Los suelos urbanos tuvieron una concentración variable de elementos mayores $\left(\mathrm{Al}_{2} \mathrm{O}_{3}, \mathrm{Fe}_{2} \mathrm{O}_{3}\right.$ y $\left.\mathrm{MnO}\right)$ y $\mathrm{Pb}$. El contenido de $\mathrm{Al}_{2} \mathrm{O}_{3}$ (9.17 a $23.47 \%), \mathrm{Fe}_{2} \mathrm{O}_{3}(5.73$ a $12.12 \%), \mathrm{MnO}(0.03$ a $0.16 \%)$ y $\mathrm{Pb}$ $\left(9\right.$ a $\left.111 \mathrm{mg} \mathrm{kg}^{-1}\right)$ fueron similares a los reportados en suelos de otras partes del mundo, tal como: $\mathrm{Al}_{2} \mathrm{O}_{3}$ entre 2 a $21 \%$ en Brasil (Souza et
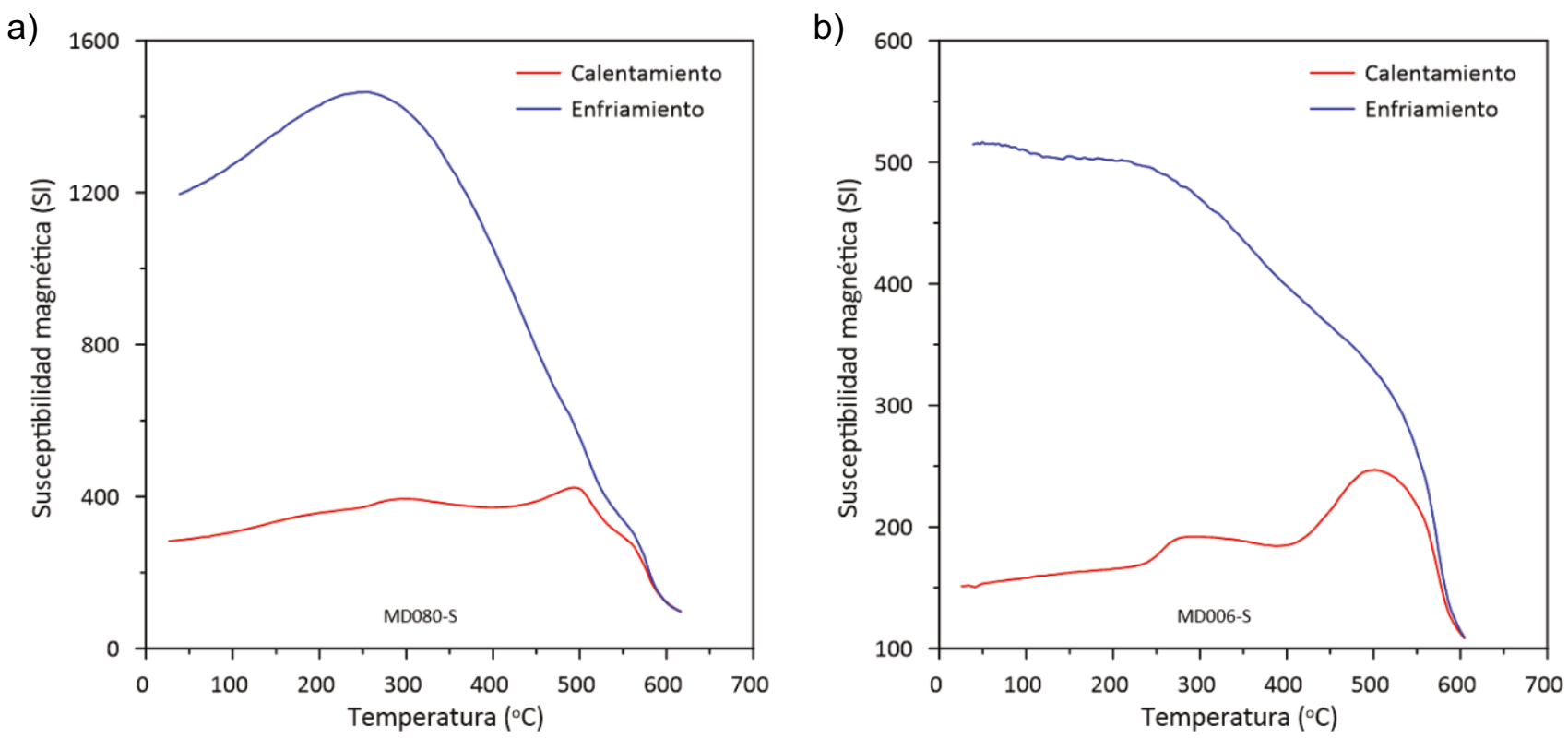

Figura 4. Curvas termomagnéticas representativas de muestras de suelos. Las variaciones de la curva indican la presencia de minerales magnéticos. 
al., 2018); $\mathrm{Fe}_{2} \mathrm{O}_{3}$ de 5.0 a $5.4 \%$ y $\mathrm{MnO}$ entre 0.8 a 1.5 para registro de suelos alrededor del mundo (de Caritat et al., 2012) y Pb entre 4.8 a $287 \mathrm{mg} \cdot \mathrm{kg}^{-1}$ para suelos agrícolas en México (Martínez-Alva et al., 2015; Rueda et al., 2011).

Se encontró que el contenido de $\mathrm{Pb}$ en los suelos del AMVA se encuentra dentro del promedio mundial para suelos tropicales no contaminados (10 a $84 \mathrm{mg} \cdot \mathrm{kg}^{-1}$ ) (Rueda et al., 2011), al igual que el contenido de Fe (7.29 a $2.10 \%$, ) y Mn (0.02 a $3.00 \%$ ) (Malavolta et al., 1962; Vásquez-Polo et al., 2014). A pesar de estar dentro los promedios mundiales, no es posible considerar un valor de referencia universal como valor de referencia, esto debido a la variabilidad de concentración que existe para cada grupo de suelo en las diferentes regiones del mundo (Horckmans et al., 2005; Fadigas et al., 2006; Brizuela y Jiménez, 2012).

\section{La comparación entre usos urbanos del suelo}

El uso de suelo EP y ABM arrojó los valores más bajos de los parámetros magnéticos, mientras que, los usos de suelo ACAM-I y ACAM-CS (Tabla 1) mostraron los valores más altos (Figuras 5a y 5b) La distribución de $\mathrm{Al}_{2} \mathrm{O}_{3}$ y $\mathrm{Fe}_{2} \mathrm{O}_{3}$ presenta un comportamiento similar entre los usos de suelo, el suelo ACMM mostró un amplio intervalo de valores, por lo que se infiere que hay sitios con enriquecimiento o escasez de $\mathrm{Al}_{2} \mathrm{O}_{3}$ y $\mathrm{Fe}_{2} \mathrm{O}_{3}$ (Figuras 5 c y $5 \mathrm{~d}$ ).

Por otra parte, el $\mathrm{MnO}$ presentó concentraciones bajas en ACAM-I y ACMM que el resto de los suelos (Figura $5 \mathrm{e}$ ). El contenido de $\mathrm{Pb}$ fue bajo en los usos de suelos EP y ABM, mientras que las concentraciones más altas fueron registradas en los usos de suelos ACMM y ACAM-I, los cuales contiene un número mayor de fuentes emisoras de partículas contaminantes (Figura 5f).

\section{Las relaciones de los parámetros magnéticos con los elementos mayores y el plomo}

Las correlaciones lineales de Pearson de los datos de las 52 muestras de suelo mostraron que el $\mathrm{Pb}$ presentó una relación estadísticamente significativa con $\chi_{\text {If }} y$ MRIS, con un nivel de confianza del $95.0 \%$ y coeficiente de correlación (CC) de 0.47 y 0.46 , respectivamente (Tabla 2 ; Tabla S2 del material suplementario). Los contenidos de $\mathrm{Al}_{2} \mathrm{O}_{3}$ y $\mathrm{MnO}$ también presentaron una relación estadísticamente significativa con $\chi_{\mathrm{lf}}$ y MRIS, la cual fue inversamente proporcional (Tabla 2).

Los modelos matemáticos lineales que se proponen para estimar la concentración de $\mathrm{Al}_{2} \mathrm{O}_{3}, \mathrm{Fe}_{2} \mathrm{O}_{3}, \mathrm{MnO}$ y $\mathrm{Pb}$ a partir de parámetros magnéticos en suelos de Medellín, se presentan en la Tabla 3.

Todos los modelos matemáticos muestran un $\mathrm{CC}>|0.30|$, valor de $\mathrm{R}^{2}>17.6$ y valor de $\mathrm{p}<0.05$, lo que indica que existe una relación estadísticamente significativa entre parámetros magnéticos, concentración de elementos mayores y concentración de $\mathrm{Pb}$ con un nivel de confianza del $95.0 \%$, por lo que es posible estimar la concentración de elementos mayores y concentración de $\mathrm{Pb}$ por medio de los modelos de regresión lineal simple para los suelos de Medellín.

La concentración de $\mathrm{Al}_{2} \mathrm{O}_{3}, \mathrm{Fe}_{2} \mathrm{O}_{3}$ y $\mathrm{MnO}$ presenta un comportamiento inversamente proporcional con las mediciones magnéticas. La concentración de $\mathrm{Al}_{2} \mathrm{O}_{3}$ y $\mathrm{MnO}$ en el suelo proporciona un comportamiento no magnético en el suelo; dado que estos elementos son abundantes en el suelo, se interpreta que los usos de suelo EP y ABM son posiblemente los valores naturales o de referencia del suelo. El contenido de $\mathrm{Fe}_{2} \mathrm{O}_{3}$ mostró un comportamiento inversamente proporcional con las mediciones magnéticas, comportamiento que puede ser atribuido al comportamiento antiferromagnético que presentan los óxidos de $\mathrm{Fe}$, los cuales se forman por el intemperismo. En Colombia, las altas temperatura y la fuerte precipitación pluvial en los suelos tropicales provocan alteración de los minerales magnéticos que modifican su comportamiento fisicoquímico (Maher, 1986; Acevedo-Sandoval et al., 2004; Ghul, 2017).
Las clases de contaminación por elementos mayores y plomo

El contenido de $\mathrm{Pb}$ fue empleado para identificar el uso de suelo con las concentraciones más bajas y determinar un valor de referencia para $\mathrm{Al}_{2} \mathrm{O}_{3}, \mathrm{Fe}_{2} \mathrm{O}_{3}, \mathrm{MnO}$ y $\mathrm{Pb}$ (Tabla 4), ya que no existe una norma oficial para estos elementos en el área de estudio. Los usos de suelo de Medellín de tipo ABM y EP presentaron las concentraciones más bajas de $\mathrm{Pb}$, muy probablemente debido a la escasez de fuentes contaminantes (emisiones vehiculares e industriales). Por otra parte, los usos de suelo ACMM y ACAM, que tienen mayores fuentes de contaminación (tráfico vehicular y presencia de industrias), muestran una mayor concentración de $\mathrm{Pb}$, pero menor concentraciones de $\mathrm{Al}_{2} \mathrm{O}_{3}, \mathrm{Fe}_{2} \mathrm{O}_{3}$ y $\mathrm{MnO}$ (Tabla 4 y Figura 5).

El valor de la media del uso de suelo EP se utilizó como definición del valor de referencia, mientras que los niveles de concentración se definieron en particiones regulares, entre el valor de referencia y el valor de la mediana más alto. Esto se debe a que los valores de las concentraciones entre los diferentes usos de suelo se agrupan en intervalos pequeños. En algunos casos, el valor más alto de la mediana del uso de suelo es menor al doble de la concentración, por lo que calcular un factor de concentración no resulta tan relevante. Los límites correspondientes para los parámetros magnéticos se determinaron por medio del modelo de regresión lineal encontrado en este estudio. Los resultados se muestran en la Tabla 4.

\section{El análisis espacial}

Los semivariogramas de $\chi, \mathrm{Al}_{2} \mathrm{O}_{3}, \mathrm{Fe}_{2} \mathrm{O}_{3}, \mathrm{MnO}$ fueron ajustados con modelos teóricos de tipo exponencial. Los parámetros de los semivariogramas fueron: nugget $<0.76$, varianza estructural $(\mathrm{C} /(\mathrm{C}+\mathrm{Co}))$ entre 50.0 a $98.6 \%$ con $\mathrm{R}^{2}$ entre 0.72 a 1.00 y una correlación espacial entre 1.125 y $8.310 \mathrm{~km}$. Los semivariogramas de MRIS y $\mathrm{Pb}$ fueron ajustados con un modelo esférico, en donde los parámetros del variograma fueron: nugget de 0.25 y 0.12 , varianza estructural de 50.1 y $57.7 \%$ con $\mathrm{R}^{2}$ de 0.93 y 0.95 y correlación espacial de 4.74 y $5.38 \mathrm{~km}$, respectivamente (Tabla 5).

Los semivariogramas de $\chi_{\mathrm{lf}}$, MRIS y concentración de $\mathrm{Pb}$ mostraron valores de varianza estructural $>50$, rango $>3.00 \mathrm{~km}$ y CC de validación cruzada $>30$. Con los cuales se obtuvieron modelos que describen con mejor precisión la distribución espacial del material magnético (Figuras 6 y 7) y la concentración de Pb (Figura 8) en la zona urbana de Medellín, Colombia.

En los mapas de distribución de $\chi_{\mathrm{lf}}$, MRIS y concentración de

Tabla 2. Coeficientes de correlación de Pearson entre los parámetros magnéticos y la concentración de elementos mayores y plomo.

\begin{tabular}{lcccccc}
\hline $\mathbf{n = 5 2}$ & $\chi_{\text {If }}$ & MRIS & $\mathbf{P b}$ & $\mathrm{Al}_{2} \mathrm{O}_{3}$ & $\mathbf{M n O}$ & $\mathrm{Fe}_{2} \mathrm{O}_{3}$ \\
\hline$\chi_{\text {lf }}$ & 1.00 & & & & & \\
& & & & & & \\
$\mathrm{MRIS}$ & $\mathbf{0 . 9 7}$ & 1.00 & & & & \\
& $\mathbf{0 . 0 0}$ & & & & & \\
$\mathrm{Pb}$ & $\mathbf{0 . 4 7}$ & $\mathbf{0 . 4 6}$ & 1.00 & & & \\
& $\mathbf{0 . 0 0}$ & $\mathbf{0 . 0 0}$ & & & & \\
$\mathrm{Al}_{2} \mathrm{O}_{3}$ & $\mathbf{- 0 . 3 7}$ & $\mathbf{- 0 . 4 2}$ & -0.20 & 1.00 & & \\
& $\mathbf{0 . 0 1}$ & $\mathbf{0 . 0 0}$ & 0.15 & & & \\
$\mathrm{MnO}$ & $\mathbf{- 0 . 3 0}$ & -0.27 & $\mathbf{- 0 . 4 0}$ & 0.12 & 1.00 & \\
& $\mathbf{0 . 0 3}$ & 0.05 & $\mathbf{0 . 0 0}$ & 0.41 & & \\
$\mathrm{Fe}_{2} \mathrm{O}_{3}$ & -0.18 & -0.25 & $\mathbf{- 0 . 3 8}$ & $\mathbf{0 . 5 1}$ & $\mathbf{0 . 3 9}$ & 1.00 \\
& 0.21 & 0.08 & $\mathbf{0 . 0 1}$ & $\mathbf{0 . 0 0}$ & $\mathbf{0 . 0 0}$ & \\
\hline
\end{tabular}

$\chi_{\text {If: }}$ Susceptibilidad específica; MRIS: magnetización remanente isotérmica de saturación. 

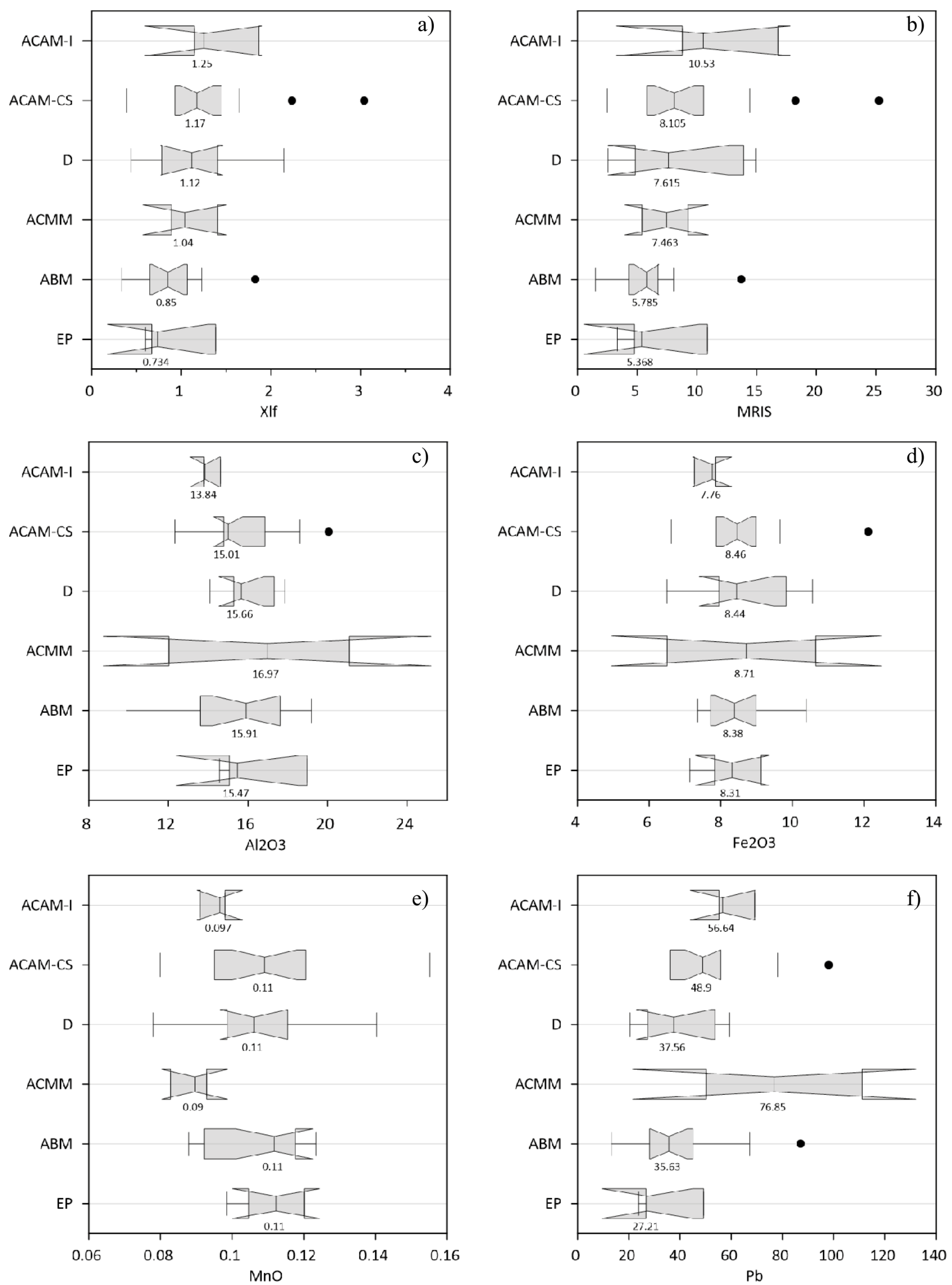

Figura 5. Gráfica de caja y bigotes para resultados de concentración de elementos y parámetros magnéticos por usos de suelo: Industrial (ACAM-I), Comercio y servicios (ACAM-CS), Dotacional (D), Áreas y corredores de media mixtura (ACMM), Áreas de baja mixtura o residenciales (ABM) y Espacio público (EP). 
Tabla 3. Modelos matemáticos para estimar la concentración de elementos mayores y plomo a partir de parámetros magnéticos.

\begin{tabular}{|c|c|c|c|c|}
\hline Parámetros & $\mathrm{CC}$ & $\mathbf{R}^{2}$ & Valor p & Modelo \\
\hline$\chi_{\text {If }}$ vs $\mathrm{Al}_{2} \mathrm{O}_{3}$ & -0.37 & 13.6 & 0.01 & $\mathrm{Al}_{2} \mathrm{O}_{3}=17.38-1.56^{*} \chi_{\mathrm{lf}}$ \\
\hline$\chi_{\text {lf }}$ vs $\mathrm{Fe}_{2} \mathrm{O}_{3}$ & -0.31 & 9.6 & 0.03 & $\mathrm{Fe}_{2} \mathrm{O}_{3}=8.88-0.64^{*} \chi_{\mathrm{lf}}$ \\
\hline$\chi_{\mathrm{lf}}$ vs $\mathrm{MnO}$ & -0.34 & 11.9 & 0.01 & $\mathrm{MnO}=0.12-0.012^{\star} \chi_{\mathrm{lf}}$ \\
\hline$\chi_{\mathrm{lf}} \mathrm{vs} \mathrm{Pb}$ & 0.47 & 22.5 & 0.00 & $\mathrm{~Pb}=23.58+19.68^{\star} \chi_{\mathrm{lf}}$ \\
\hline MRIS vs $\mathrm{Al}_{2} \mathrm{O}_{3}$ & -0.42 & 17.3 & 0.00 & $\mathrm{Al}_{2} \mathrm{O}_{3}=17.25-0.20^{\star}$ MRIS \\
\hline MRIS vs $\mathrm{Fe}_{2} \mathrm{O}_{3}$ & -0.36 & 12.7 & 0.01 & $\mathrm{Fe}_{2} \mathrm{O}_{3}=8.82-0.083^{\star} \mathrm{MRIS}$ \\
\hline MRIS vs MnO & -0.33 & 10.8 & 0.02 & $\mathrm{MnO}=0.115-0.001^{\star} \mathrm{MRIS}$ \\
\hline MRIS vs $\mathrm{Pb}$ & 0.46 & 21.1 & 0.00 & $\mathrm{~Pb}=28.084+2.177^{\star} \mathrm{MRIS}$ \\
\hline
\end{tabular}

CC: Coeficiente de correlación; $\mathrm{R}^{2}$ : Coeficiente de determinación.

$\mathrm{Pb}$ se observaron similitudes en las áreas de mayor acumulación, la cual es más semejante en la zona central del área metropolitana. Por otra parte, se observó una acumulación de materiales magnéticos en la zona periurbana al norte y sur donde es probable que estas áreas puedan contener concentraciones de $\mathrm{Pb}$ altas, sin embargo, esto no se evidenció en este estudio (Figuras 6 y 7 ). Estas zonas son susceptibles a convertirse en zonas de acumulación de $\mathrm{Pb}$ y otros elementos; la forma del relieve donde se construyó el AMVA es un factor importante en la acumulación de material magnético y concentración de $\mathrm{Pb}$ y que se presentó en las partes bajas del valle (Figuras 6, 7 y 8 ).

Las áreas de acumulación de material magnético ( $\chi_{\text {If }}$ y MIRS) de este estudio fueron similares a la reportadas por Mejía-Echeverry et al. (2018) a partir del análisis de muestras botánicas de la especie Tillandsia recurvata en 2016, aunque en este trabajo, las áreas de mayor acumulación muestran una superficie más amplia a la que la que reportan esos autores.

El área que ocupan las zonas con bajas o sin alteración fue de 114 a $140 \mathrm{~km}^{2}$, aproximadamente. Por otra parte, el área de las zonas de alta o muy alta alteración en la concentración de elementos fue de 23 a $46 \mathrm{~km}^{2}$ (Tabla 6).

La ubicación geográfica de las áreas de mayor acumulación de material obtenidas en este estudio es muy similar con lo reportado por Mejía-Echeverry et al., (2018), en cambio, las áreas de menor acumulación son diferentes. Esta diferencia se debe muy probablemente al tipo de muestra, ya que el material botánico tiene un ciclo biológico que pude alterar la concentración de los minerales, aunque el suelo también puede verse afectado por remociones de tipo antrópico o por fenómenos naturales. Sin embargo, en ambos estudios se prueba la eficacia de determinar la concentración de ciertos metales pesados o mayores por medio de parámetros magnéticos con cierto grado de precisión y con resultados geográficos muy similares. En este estudio,
Tabla 4. Clases para los elementos mayores, plomo y parámetros magnéticos.

\begin{tabular}{lccccc}
\hline Parámetro & \multicolumn{5}{c}{ Nivel } \\
\cline { 2 - 6 } & $\begin{array}{c}\text { Valor } \\
\text { referencia }\end{array}$ & Bajo & Medio & Alto & Muy alto \\
\hline $\mathrm{Pb}$ & 38 & 48 & 57 & 67 & 76 \\
$\chi_{\mathrm{If}}$ & 1.0 & 1.1 & 1.2 & 1.3 & 1.5 \\
$\mathrm{MRIS}$ & 7.2 & 8.1 & 9.0 & 9.9 & 10.9 \\
$\mathrm{Al}_{2} \mathrm{O}_{3}{ }^{*}$ & 17.44 & 16.69 & 15.94 & 15.19 & 13.84 \\
$\chi_{\mathrm{lf}}$ & 0.95 & 1.01 & 1.08 & 1.14 & 1.26 \\
$\mathrm{MRIS}$ & 6.35 & 6.99 & 7.64 & 8.28 & 9.45 \\
$\mathrm{Fe}_{2} \mathrm{O}_{3}{ }^{*}$ & 9.22 & 8.86 & 8.49 & 8.13 & 7.76 \\
$\chi_{\mathrm{lf}}$ & 0.90 & 0.95 & 1.01 & 1.06 & 1.12 \\
$\mathrm{MRIS}$ & 5.99 & 6.55 & 7.10 & 7.66 & 8.23 \\
$\mathrm{MnO}^{*}$ & 0.12 & 0.12 & 0.11 & 0.10 & 0.09 \\
$\chi_{\mathrm{lf}}$ & 0.89 & 0.97 & 1.06 & 1.14 & 1.23 \\
$\mathrm{MRIS}^{*}$ & 6.09 & 6.79 & 7.49 & 8.19 & 8.89 \\
\hline
\end{tabular}

${ }^{\star}$ Comportamiento inversamente proporcional. $\chi_{\text {lf: }}$ Susceptibilidad específica $\left(\mu \mathrm{m}^{3} \cdot \mathrm{kg}^{-1}\right)$; MRIS: magnetización remanente isotérmica de saturación $\left(\mathrm{mAm}^{2} \cdot \mathrm{kg}^{-1}\right)$.

la precisión de determinar elementos mayores $\left(\mathrm{Al}_{2} \mathrm{O}_{3}, \mathrm{Fe}_{2} \mathrm{O}_{3}, \mathrm{MnO}\right)$ y concentración de $\mathrm{Pb}$ usando los parámetros magnéticos ( $\chi_{\mathrm{lf}} \mathrm{o}$ MRIS) fue de $63 \%$

Por otra parte, un estudio para determinar las variaciones temporales aún no es factible, debido principalmente a la escasez de datos y a la diversidad del tipo de muestras (suelo, polvo urbano, sedimentos, material botánico, etc.) que se usan para los estudios de magnetismo y su relación con la concentración de metales pesados. La homologación de valores de referencia magnéticos y de concentración de elementos (mayores o metales pesados) no es viable de forma general, por lo que para cada zona urbana o área de estudio deberá de ajustarse en función de tipo de muestra ambiental empleada y de los factores geográficos; de esta forma se podrá obtener un sistema de monitoreo confiable a través de parámetros magnéticos para esa área local.

\section{CONCLUSIONES}

Los análisis de los diferentes parámetros magnéticos del suelo urbano superficial indican que la señal magnética se debe a la presencia de minerales ferrimagnéticos de baja coercitividad de origen tanto antrópico como natural. Hay una mayor concentración de material magnético en los usos de suelo que presentan fuentes de contaminación asociados a las emisiones vehiculares y presencia de comercio e

Tabla 5. Correlación espacial para elementos mayores, plomo y parámetros magnéticos.

\begin{tabular}{llccccccc}
\hline Parámetro & Modelo & $\begin{array}{c}\text { Nugget } \\
\mathrm{C}_{0}\end{array}$ & $\begin{array}{c}\text { Sill } \\
\mathrm{C}+\mathrm{C}_{0}\end{array}$ & $\mathrm{C}_{\mathbf{l}}$ & $\begin{array}{c}\text { Varianza } \\
\text { estructural } \\
(\%)\end{array}$ & $\mathbf{R}^{2}$ & $\begin{array}{c}\text { Rango } \\
(\mathrm{km})\end{array}$ & $\begin{array}{c}\text { Validación } \\
\text { cruzada } \\
\text { CC }\end{array}$ \\
\hline$\chi_{\mathrm{lf}}$ & Exponencial & $\mathbf{0 . 0 3}$ & $\mathbf{0 . 3 6}$ & $\mathbf{0 . 3 3}$ & $\mathbf{9 1 . 7}$ & $\mathbf{0 . 9 4}$ & $\mathbf{3 . 2 1 0}$ & $\mathbf{0 . 3 6}$ \\
$\mathrm{MRIS}$ & Esférico & $\mathbf{0 . 2 4}$ & $\mathbf{0 . 4 9}$ & $\mathbf{0 . 2 4}$ & $\mathbf{5 0 . 1}$ & $\mathbf{0 . 9 3}$ & $\mathbf{4 . 7 4 0}$ & $\mathbf{0 . 3 7}$ \\
$\mathrm{Al}_{2} \mathrm{O}_{3}$ & Exponencial & 0.09 & 6.30 & 6.21 & 98.6 & 0.93 & 1.620 & 0.43 \\
$\mathrm{Fe}_{2} \mathrm{O}_{3}$ & Exponencial & 0.76 & 1.52 & 0.76 & 50.0 & 0.72 & 8.310 & 0.50 \\
$\mathrm{MnO}$ & Exponencial & $5.9 \times 10^{-5}$ & $5.1 \times 10^{-4}$ & $4.5 \times 10^{-4}$ & 88.4 & 1.00 & 1.125 & 0.02 \\
$\mathbf{P b}$ & Esférico & $\mathbf{0 . 1 2}$ & $\mathbf{0 . 2 8}$ & $\mathbf{0 . 1 6}$ & $\mathbf{5 7 . 7}$ & $\mathbf{0 . 9 5}$ & $\mathbf{5 . 3 8 0}$ & $\mathbf{0 . 8 5}$ \\
\hline
\end{tabular}

$\mathrm{C}_{1}$ : Semivarianza estructural; $\mathrm{R}^{2}$ : Coeficiente de determinación 


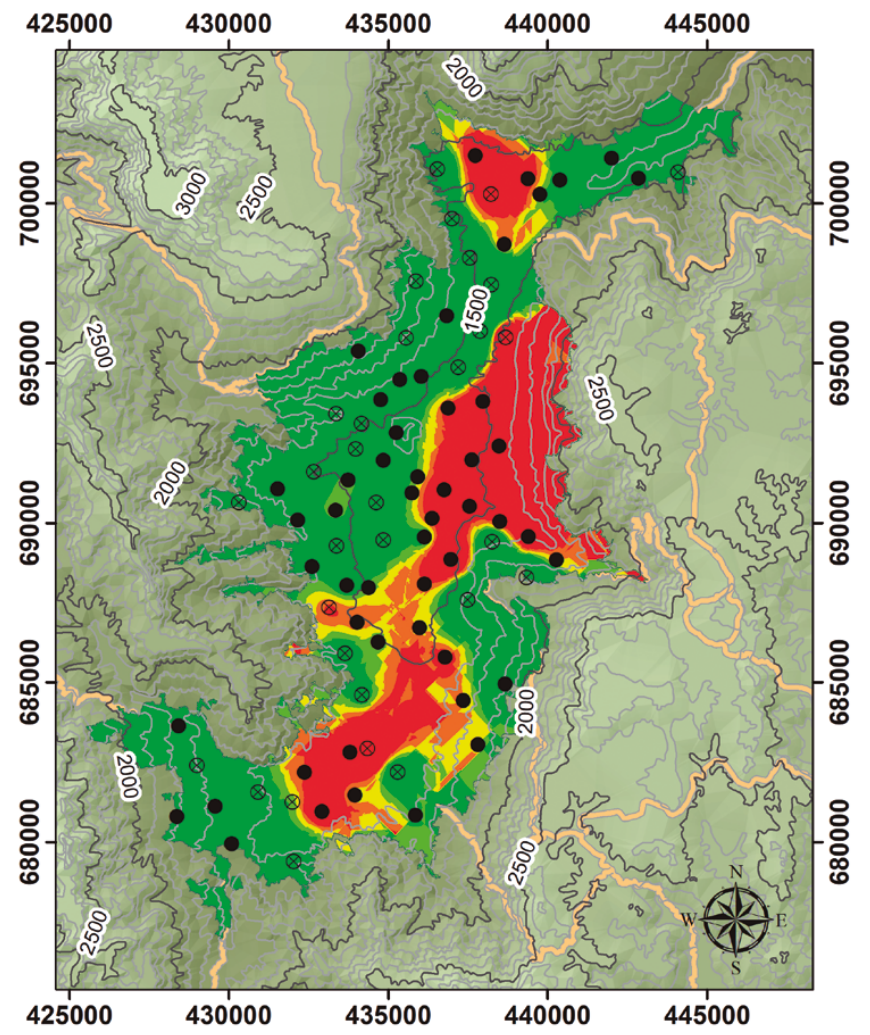

\author{
Leyenda \\ Sitio \\ - Con correlación \\ $\otimes$ Sin correlación \\ XIf \\ $10^{-6} \mathrm{~m}^{3} \mathrm{~kg}^{-1}$ \\ Valor referencia \\ Bajo \\ $\square$ Medio \\ Alto \\ Muy alto \\ Símbolos convencionales \\ Carretera \\ Límite urbano
}

Fecha de muestreo

AMVA-------------Enero de 2014

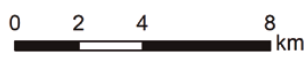

Referencia espacial

Elipsoide.......................WGS84 1984

Proyección.........Transversal Mercantor

Zona..................................18 Norte

Meridiano Central. $75^{\circ}$ Oeste

Longitud de referencia.................. $0^{\circ}$ Norte

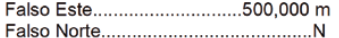

Figura 6. Distribución espacial de valores de susceptibilidad específica $\left(\chi_{\mathrm{If}}\right)$ en suelos urbanos del Área Metropolitana Valle de Aburrá, Colombia.

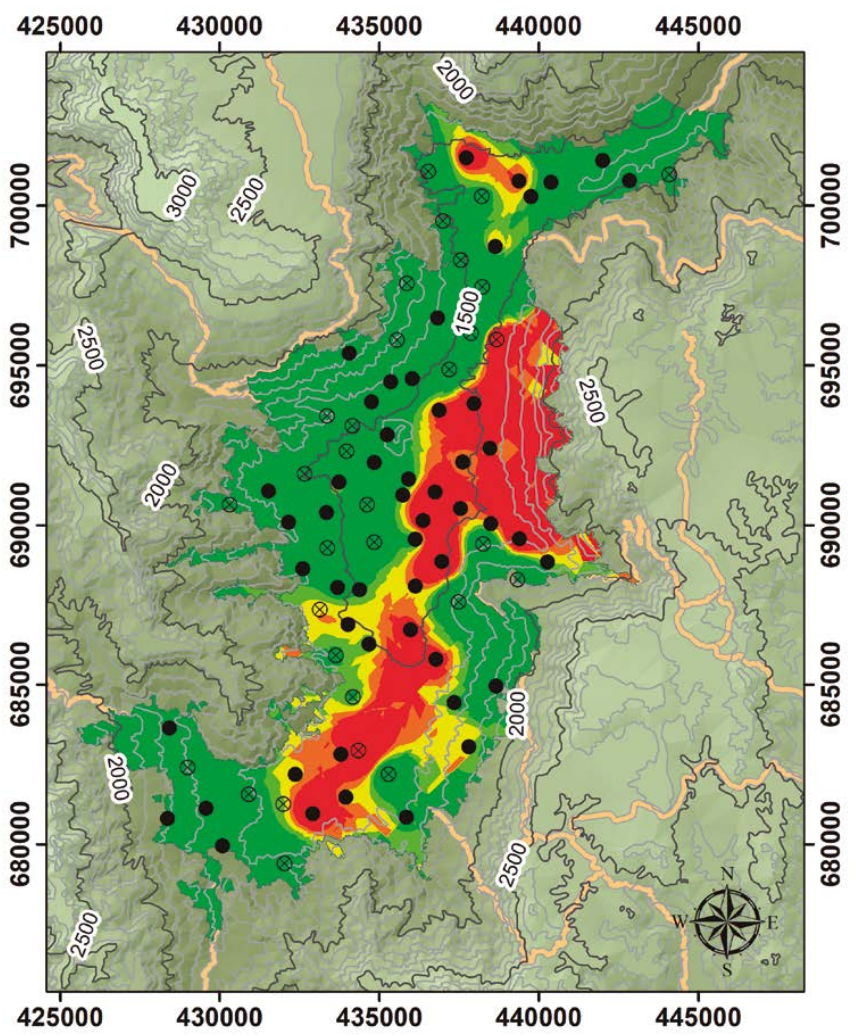

Leyenda

Sitio

- Con correlación

$\otimes$ Sin correlación

MRIS

$\mathrm{mAm}^{2} \mathbf{k g}^{-1}$

Valor referencia

Bajo

$\square$ Medio

Alto

Muy alto

Símbolos convencionales

Carretera

_ Límite urbano

Fecha de muestreo

AMVA---_-------Enero de 2014

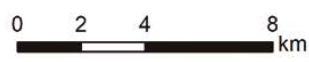

Referencia espacial

Elipsoide......................WGS84 1984 Proyección..........Transversal Mercantor Zona................................ 18 Norte

Deridiano Central $\quad . . . . . . . . W G S$ Lengitud de referenci........... Longitud de referencia............... Norte Falso Este................................500,000 m Falso Norte....

Figura 7. Distribución espacial de valores de magnetización remanente isotérmica a saturación (MRIS) en suelos urbanos del Área Metropolitana Valle de Aburrá, Colombia. 


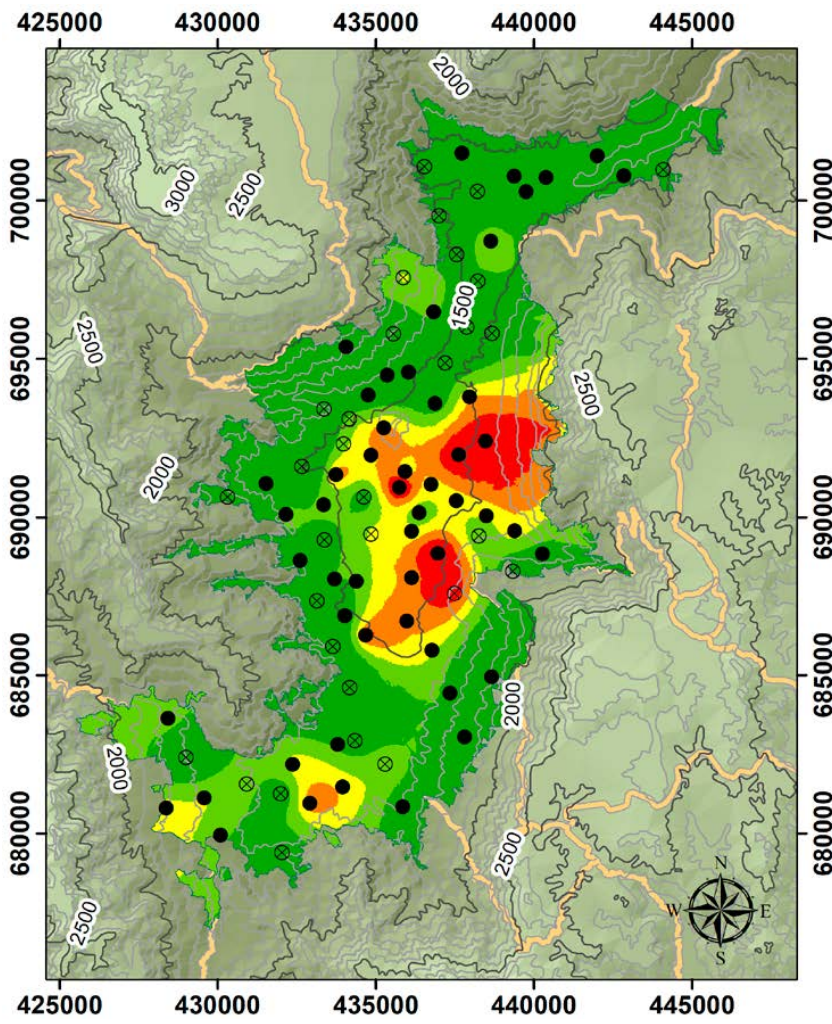

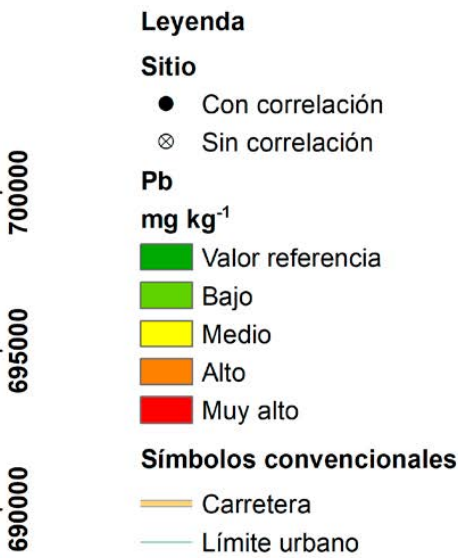

Fecha de muestreo

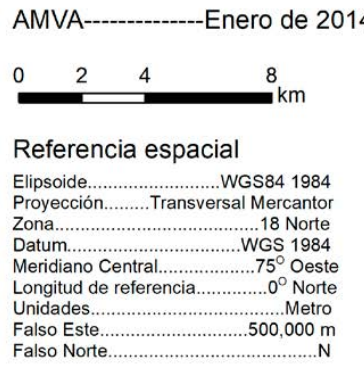

Figura 8. Concentración de $\mathrm{Pb}$ en suelos urbanos del Área Metropolitana Valle de Aburrá, Colombia.

industrias. La presencia de usos de suelo comercial e industrial incrementa el flujo y tráfico vehicular, por lo que son un factor importante en la emisión de material magnético, elementos mayores y contenido de plomo; en las áreas próximas de estos usos de suelo se observó una mayor concentración de material magnético y contenido de $\mathrm{Pb}$, la cual en algunos casos fue de más del doble o tripe que en los otros usos de suelo.

El suelo de AMVA presentó material magnético superparamagnético de origen natural en concentración media a alta, sin embargo, también hay un enriquecimiento de magnetita de origen antropogénico en casi en la totalidad de los suelos urbanos.

Las zonas residenciales y de espacio público mantienen concentraciones bajas de emisión de material antrópico, por lo que son áreas para determinar valores de referencia confiables para parámetros magnéticos, elementos mayores y concentración de $\mathrm{Pb}$.

Son preocupantes los niveles de concentración de plomo que se registraron en los suelos de la zona centro de la ciudad y las áreas pe- riurbanas que están en el norte y sur del AMVA, ya que son las áreas que concentran la mayor población y que presentan un crecimiento urbano, por lo que hay una tendencia a ser zonas de riesgo.

El uso de parámetros magnéticos puede ser usado como monitoreo proxy de elementos mayores y concentración de $\mathrm{Pb}$ en los suelos de la AMVA. Sin embargo, el uso de la metodología magnética solo es un estimador rápido y no exenta el uso de otros métodos para la evaluación más precisa para determinar la concentración de elementos químicos en el suelo. La susceptibilidad magnética y magnetización remanente tiene una efectividad del $63 \%$ para estimar la concentración de $\mathrm{Al}_{2} \mathrm{O}_{3}$, $\mathrm{Fe}_{2} \mathrm{O}_{3}, \mathrm{MnO}$ y $\mathrm{Pb}$.

Los valores de referencia encontrados para el suelo urbano y que determinan alteraciones en el contenido de elementos mayores y la concentración de $\mathrm{Pb}$ por medio de parámetros magnéticos son solo efectivos para el Valle de Aburrá, ya que cada zona geográfica contiene factores formadores de suelo distintos, además de que las actividades antrópicas complican el establecimiento de un valor de referencia.

Tabla 6. Área estimada en $\mathrm{km}^{2}$ por cada clase de concentración de plomo y parámetros magnéticos.

\begin{tabular}{|c|c|c|c|c|c|c|c|}
\hline Parámetro & & Sin aumento & Bajo & Medio & Alto & Muy alto & Total \\
\hline \multirow[t]{2}{*}{$\mathrm{Pb}$} & * & 103 & 37 & 20 & 15 & 8 & 183 \\
\hline & $* *$ & 56 & 20 & 11 & 8 & 5 & 100 \\
\hline \multirow[t]{2}{*}{$\chi_{\mathrm{lf}}$} & $*$ & 101 & 13 & 13 & 14 & 41 & 183 \\
\hline & ** & 55 & 7 & 7 & 8 & 23 & 100 \\
\hline \multirow[t]{2}{*}{ MRIS } & * & 105 & 15 & 17 & 13 & 33 & 183 \\
\hline & ** & 58 & 8 & 9 & 7 & 18 & 100 \\
\hline
\end{tabular}

*: Área estimada en $\mathrm{km}^{2} ;{ }^{* *}$ : porcentaje del área del Área Metropolitana del Valle de Aburrá; $\chi_{\mathrm{lf}}$ Susceptibilidad específica; MRIS: magnetización remanente isotérmica de saturación. 


\section{AGRADECIMIENTOS}

Al Consejo Nacional de Ciencia y Tecnología (CONACyT) por el apoyo económico al proyecto clave $\mathrm{CB}-283135$. A los árbitros por el tiempo y comentarios hechos durante la revisión de este manuscrito.

\section{MATERIAL SUPLEMENTARIO}

Las Tablas S1 y S2 pueden ser descargados del portal web de la revista $<$ http://rmcg.unam.mx/>, en la versión html de este artículo.

\section{REFERENCIAS}

Acevedo-Sandoval, O., Ortiz-Hernández, E., Cruz-Sánchez, M., Cruz-Chávez, E., 2004, El papel de óxidos de hierro en suelos: Terra Latinoamericana, 22(4), 485-497.

Alcaldía de Medellín, 2011, Medellín y su población, en Documento Técnico de Soporte POT [ACUERDO 46/2006], Primera Parte: Generalidades: Medellín, Colombia, Alcaldía de Medellín, 4, 8 pp. acceso libre, disponible en <https://www.medellin.gov.co/irj/go/km/docs/wpccontent/Sites/ Subportal del Ciudadano/Plan de Desarrollo/Secciones/Información General/Documentos/POT/medellinPoblacion.pdf $>$, consulta: 20 junio 2020.

Brizuela J., Jiménez Y., 2012, Metodologías aplicadas para el establecimiento de los niveles de referencia para metales pesados en la evaluación de la contaminación en suelos: Avances en Química, 7(2), 101-109.

Brus, D.J., Lamé, F.P.J., Nieuwenhuis, R.H., 2009, National baseline survey of soil quality in the Netherlands: Environmental Pollution, 157, 2043-2052. DOI: 10.1016/j.envpol.2009.02.028.

Cairney, T., Hobson, D., 1998, Contaminated Land: Problems and Solutions: London, U.K., Taylor \& Francis, 369 pp., <http://www.crcnetbase.com/ doi/book/10.4324/9780203477625>, consultado: junio 2015.

Cejudo-Ruíz, R., Bautista, F., Quintana, P., Delgado-Carranza, M. del C., Aguilar, D., Goguitchaichvili, A., Morales-Contreras, J.J., 2015, Correlación entre elementos potencialmente tóxicos y propiedades magnéticas en suelos de la Ciudad de México para la identificación de sitios contaminados: definición de umbrales magnéticos: Revista Mexicana de Ciencias Geológicas, 32,(1), 50-61.

CGM (Contraloría General de Medellín), 2015, Estado de los Recursos Naturales y del Ambiente del Municipio de Medellín 2014: Medellín, Colombia, Contraloría General de Medellín, 264 pp.

Chilès, J.-P., Delfiner, P., 2012, Geostatistics: Modeling Spatial Uncertainty: Hoboken, NJ, USA, John Wiley \& Sons, Inc., 734 pp. http://doi.wiley. com/10.1002/9781118136188.

Dearing, J.A., 1999, Magnetic susceptibility, en Walden, J., Oldfield, F., Smith, J. (eds.), Environmental magnetism: a practical guide: London, Quaternary Research Association, Technical guide No. 6, 35-62.

de Caritat P., Reimann C., NGSA Project Team, GEMAS Project Team., 2012, Comparing results from two continental geochemical surveys to world soil composition and deriving Predicted Empirical Global Soil (PEGS2) reference values: Earth and Planetary Science Letter, 319-320, 269-276, DOI:10.1016/j.epsl.2011.12.033.

DAPM (Departamento Administrativo de Planeación Municipal), 2014, Acuerdo 048 de 2014 "Por medio del cual se adopta la revisión y ajuste de largo plazo del Plan de Ordenamiento Territorial del Municipio de Medellín y se dictan otras disposiciones complementarias": Gaceta Oficial, p. 877.

ESRI (Environmental Systems Research Institute), 2005, ArcGIS Desktop, ver. 9.1: Redlands, USA, Environmental Systems Research Institute, Inc., programa informático.

Evans, M.E., Heller, F., 2003, Environmental magnetism: principles and applications of enviromagnetics: San Diego, California, Academic Press, $317 \mathrm{pp}$.

Fadigas, F.D., Sobrinho, N., Mazur, N., dos Anjos L.H., 2006, Estimation of reference values for cadmium, cobalt, chromium, copper, nickel, lead, and zinc in Brazilian soils: Communications in Soil Science and Plant Analysis, 37: 945-959, doi: 10.1080/00103620600583885.

Gamma Design Software, 2014, GS+ Geostatistics for the Environmental Sciences, ver. 10.0: Plainwell, Michigan, USA, Gamma Design Software, LLC, programa informático.

Ghul, E., 2017, Colombia. Bosquejo de su geografía tropical, vol 2: Bogotá, Colombia, Universidad de los Andes, 555 pp.

Hanesch, M., Rantitsch, G., Hemetsberger, S., Scholger, R., 2007, Lithological and pedological influences on the magnetic susceptibility of soil: Their consideration in magnetic pollution mapping: Science of The Total Environment, 382, 351-363, doi:10.1016/j.scitotenv.2007.04.007.

Harrison, R.M., 2006, An Introduction to Pollution Science: Cambridge, United Kingdom, Royal Society of Chemistry, $345 \mathrm{pp}$.

Heller, F., Strzyszcz, Z., Magiera, T., 1998, Magnetic record of industrial pollution in forest soils of Upper Silesia, Poland: Journal of Geophysical Research Solid Earth, 103, 17767-17774, doi:10.1029/98JB01667.

Hernández, A.J., Alexis, S., Pastor, J., 2007, Soil degradation in the tropical forests of the Dominican Republic's Pedernales province in relation to heavy metal contents: Science of The Total Environment, 378, 36-41, doi: 10.1016/j.scitotenv.2007.01.029.

Horckmans, L., Swennen, R., Deckers, J., Maquil, R., 2005, Local background concentrations of trace elements in soils: a case study in the Grand Duchy of Luxembourg: CATENA, 59, 279-304. https://doi.org/10.1016/j. catena.2004.09.004.

Isaaks, E.H., Srivastava, R.M., 1990, An Introduction to Applied Geostatistics: New York, USA, Oxford University Press, 592 pp.

Jordanova, N.V., Jordanova, D.V., Veneva, L., Yorova, K., Petrovsky, E., 2003 Magnetic Response of Soils and Vegetation to Heavy Metal Pollution A Case Study: Environmental Science \& Technology, 37, 4417-4424. doi:10.1021/es0200645.

Jordanova, N., Jordanova, D., Tsacheva, T., 2008, Application of magnetometry for delineation of anthropogenic pollution in areas covered by various soil types: Geoderma, 144, 557-571, doi:10.1016/j.geoderma.2008.01.021.

Kabata-Pendias, A., 2010, Trace Elements in Soils and Plants, Fourth Edition: Boca Raton, FL, U.S.A., CRC Press, 548 pp.

Karimi, R., Ayoubi, S., Jalalian, A., Sheikh-Hosseini, A.R., Afyuni, M., 2011, Relationships between magnetic susceptibility and heavy metals in urban topsoils in the arid region of Isfahan, central Iran: Journal of Applied Geophysics, 74, 1-7, doi:10.1016/j.jappgeo.2011.02.009.

Lince-Prada M.F., Rave-Herrera, B.E. (coord.), 2010, Atlas Área Metropolitana del Valle de Aburrá: Medellín, Colombia, editado por Área Metropolitana del Valle de Aburrá, $46 \mathrm{pp}$.

Liu, D., Ma, J., Sun, Y., Li, Y., 2016, Spatial distribution of soil magnetic susceptibility and correlation with heavy metal pollution in Kaifeng City, China: CATENA, 139, 53-60. doi:10.1016/j.catena.2015.11.004.

Liu, Q., Roberts, A.P., Larrasoaña, J.C., Banerjee, S.K., Guyodo, Y., Tauxe, L., Oldfield, F., 2012, Environmental magnetism: Principles and applications: Reviews of Geophysics, 1-50, https://doi.org/10.1029/2012RG000393.

Londoño-Franco, F., Londoño-Muñoz, P, Muñoz-García, F., 2016, Los riesgos de los metales pesados en la salud humana y animal: Biotecnología en el Sector Agropecuario y Agroindustrial, 14,(2), 145-153, DOI:10.18684/ BSAA(14)145-153.

Lozano, R., Bernal J., 2005, Characterization of a new set of eight geochemical reference materials for XRF major and trace element analysis: Revista Mexicana de Ciencias Geológicas, 22(3), 329-344.

Lu, S.G., Bai, S.Q., 2006, Study on the correlation of magnetic properties and heavy metals content in urban soils of Hangzhou City, China: Journal of Applied Geophysics, 60, 1-12, doi:10.1016/j.jappgeo.2005.11.002.

Maher, B.A., 1986, Characterisation of soils by mineral magnetic measurements: Physics of the Earth and Planetary Interiors, 42, 76-92, DOI:10.1016/ S0031-9201(86)80010-3.

Malavolta, E., Haag, H.P., Melo, F., Brasil, M.O.C., 1962, On the mineral nutrition of some tropical crops: Berna, Suiza, International Potash Institute, $155 \mathrm{pp}$.

Martínez-Alva G., Gutiérrez-Ruiz, M.E., Martínez-Campos, A.R., VillalobosPietrini, R., Arteaga-Reyes, T.T., 2015, Concentración total y geodisponible de elementos potencialmente tóxicos en suelos volcánicos con uso agrícola del Nevado de Toluca, México: Revista Internacional de Contaminación Ambiental, 31(2), 113-125.

Mejía-Echeverry D., Chaparro, M.A.E., Duque-Trujillo, J.F., Chaparro, M.A.E., 
Castañeda-Miranda, A.G., 2018, Magnetic Biomonitoring as a Tool for Assessment of Air Pollution Patterns in a Tropical Valley Using Tillandsia sp: Atmosphere, 9(7), 283, doi:10.3390/atmos9070283.

Micó, C., Recatalá, L., Peris, M., Sánchez, J., 2006, Assessing heavy metal sources in agricultural soils of an European Mediterranean area by multivariate analysis: Chemosphere, $65,863-872$, doi.org/10.1016/j. chemosphere.2006.03.016.

MMA (Ministerio del Medio Ambiente Gobierno de Chile), 2012, Guía metodológica para la gestión del suelo con potencial presencia de contaminantes: Chile, Santiago de Chile, Ministerio del Medio Ambiente Santiago de Chile, Gobierno de Chile y Fundación Chile, 147 pp.

Oliver, M.A., Webster, R., 2014, A tutorial guide to geostatistics: Computing and modelling variograms and kriging: CATENA, 113, 56-69, doi:10.1016/j. catena.2013.09.006.

Oliver, M.A., Webster, R., 2015, Basic Steps in Geostatistics: The Variogram and Kriging: New York, USA, Springer International Publishing, $100 \mathrm{pp}$

Pérez-Martínez, I., Romero, F.M., 2015, Uso de parámetros indirectos para la evaluación de la contaminación de suelos por metales pesados en una zona minera de San Luis Potosí, México: Boletín de la Sociedad Geológica Mexicana, 67, 1-12, DOI:10.18268/BSGM2015v67n1a1.

Rodríguez-Eugenio, N., McLaughlin, M., Pennock, D., 2019, La contaminación del suelo: una realidad oculta: Roma, Organización de las Naciones Unidas para la Alimentación y la agricultura (FAO). 144 pp.

Rueda-Saa, G., Rodríguez-Victoria, J.A., Madriñán-Molina, R., 2011, Metodologías para establecer valores de referencia de metales pesados en suelos agrícolas: perspectivas para Colombia: Acta Agronómica, 60, (3), 203-218, <http://www.scielo.org.co/pdf/acag/v60n3/v60n3a01.pdf >.

Sánchez-Duque, A., Bautista, F., Goguitchaichvili, A., Cejudo-Ruiz, R., Reyes-López, J.A., Solís-Domínguez, F.A., Morales-Contreras, J.J., 2015, Evaluación de la contaminación ambiental a partir del aumento magnético en polvos urbanos. Caso de estudio en la ciudad de Mexicali, México: Revista Mexicana de Ciencias Geológicas, 32(3), 501-513.

SEMARNAT (Secretaría de Medio Ambiente y Recursos Naturales), 2002, Norma Oficial Mexicana, NOM-021- RECNAT-2000, Que establece las especificaciones de fertilidad, salinidad y clasificación de suelos. Estudios, muestreo y análisis: Ciudad de México, México, Diario Oficial de la Federación, 73 pp.

Spiteri, C., Kalinski, V., Rösler, W., Hoffmann, V., Appel, E., 2005, Magnetic screening of a pollution hotspot in the Lausitz area, Eastern Germany: correlation analysis between magnetic proxies and heavy metal contamination in soils: Environmental Geology, 49, 1-9, doi:10.1007/ s00254-005-1271-9.
Souza, J.J.L.L., Fontes, M.P.F., Gilkes, R., da Costa, L.M., Oliveira, T.S., 2018, Geochemical signature of Amazonian tropical rainforest soils: Revista Brasileira de Ciência do Solo, 42, 1-18. https://doi.org/10.1590/180696 $57 \mathrm{rbcs} 20170192$.

Tauxe, L., Banerjee, S.K., Butler, R.F., Van der Voo, R., 2018, Essentials of Paleomagnetism, 5th Web Edition, acceso libre en <https://earthref.org/ MagIC/books/Tauxe/Essentials/>, consulta: 17 de junio de 2020.

Thompson, R., Oldfield, F., 1986, Environmental Magnetism: Londres, Allen and Unwin, $227 \mathrm{pp}$.

UN/Medellín (Universidad Nacional de Colombia - Sede Medellín), AMVA (Área Metropolitana del Valle de Aburrá), CORNARE (Corporación Autónoma Regional de las Cuencas de los Ríos Negro y Nare), CORANTIOQUIA (Corporación Autónoma Regional del Centro de Antioquia), 2007, Recurso Suelo, in Plan de Ordenación y Manejo de la Cuenca del Río Aburrá (POMCA), Tomo II Diagnóstico, Volumen 1 Subsistema Abiótico: Medellín, Colombia, editado por Área Metropolitana del Valle de Aburrá, 195 pp.

Vásquez-Polo, J.R., Macías-Vázquez, F., Menjivar-Flores, J.C., 2014, Formas de hierro y aluminio en suelos con diferentes usos en la zona norte del departamento del Magdalena, Colombia: Acta Agronómica, 63(4), 352$360,<$ https://revistas.unal.edu.co/index.php/acta_agronomica/article/ view/42038>.

Wang, X.S., 2013, Heavy metal pollution in urban topsoils: mineralogical analyses and magnetic characterization: Environmental Earth Sciences, 70, 3155-3161, DOI:10.1007/s12665-013-2380-5.

Webster, R., Oliver, M.A., 1990, Statistical methods in soil and land resource survey: New York, EE.UU., Oxford University Press, 316 pp.

Xenemetrix, 2020, X-Calibur EDXRF Bench-Top Spectrometer Multielement Analyzer, Brochure: Israel, disponible en $<\mathrm{https}$ ://xenemetrix. com/?product $=\mathrm{x}$-calibur $>, 4 \mathrm{pp}$, consultado el 17 de junio de 2020 .

Xia, D., Wang, B., Yu, Y., Jia, J., Nie, Y., Wang, X., Xu, S., 2014, Combination of magnetic parameters and heavy metals to discriminate soil-contamination sources in Yinchuan - A typical oasis city of Northwestern China: Science of The Total Environment, 485-486, 83-92, doi:10.1016/j. scitotenv.2014.03.070.

Manuscrito recibido: junio18, 2020

Manuscrito corregido recibido: septiembre 17, 2020

Manuscrito aceptado: septiembre 19, 2020 